\title{
Introduction of the one-body correlation operator in the unitary-model-operator approach
}

\author{
Takayuki Miyagi, ${ }^{1}$ Takashi Abe, ${ }^{2}$ Ryoji Okamoto,${ }^{3}$ and Takaharu Otsuka ${ }^{4,2,5,6}$ \\ ${ }^{1}$ Center for Nuclear Study, University of Tokyo, Hongo, Tokyo 113-0033, Japan \\ ${ }^{2}$ Department of Physics, University of Tokyo, Hongo, Tokyo 113-0033, Japan \\ ${ }^{3}$ Senior Academy, Kyushu Institute of Technology, Tobata, Kitakyushu 804-0015, Japan \\ ${ }^{4}$ RIKEN Nishina Center, 2-1 Hirosawa, Wako, Saitama 351-0198, Japan \\ ${ }^{5}$ National Superconducting Cyclotron Laboratory, Michigan State University, East Lansing, Michigan 48824, USA \\ ${ }^{6}$ Instituut voor Kern- en Stralingsfysica, KU Leuven, B-3001 Leuven, Belgium
}

(Received 2 August 2017; published 15 November 2017)

\begin{abstract}
In the earlier unitary-model-operator approach (UMOA), one-body correlations have been taken into account approximately by the diagonalization of unitary-transformed Hamiltonians in the $0 \mathrm{p} 0 \mathrm{~h}$ and $1 \mathrm{p} 1 \mathrm{~h}$ space. With this prescription, the dependence of the harmonic-oscillator energy $(\hbar \omega)$ on calculated observables is not negligible even at larger model spaces. In the present work, we explicitly introduce the one-body correlation operator so that it optimizes the single-particle basis states and then reduces the $\hbar \omega$ dependence. For an actual demonstration, we calculate the energy and radius for the ${ }^{4} \mathrm{He}$ ground state with the softened nucleon-nucleon $(N N)$ interactions from Argonne v18 (AV18) and chiral effective field theory ( $\chi$ EFT) up to the next-to-next-to-next leading order $\left(\mathrm{N}^{3} \mathrm{LO}\right)$. As a result, we obtain practically $\hbar \omega$-free results at sufficiently large model spaces. The present results are reasonably close to those by the other $a b$ initio calculations with the same $N N$ interactions. This methodological development enables more systematic analysis of calculation results in the UMOA. We also discuss qualitatively the origin of the $\hbar \omega$ dependence on calculated observables in a somewhat simplified way.
\end{abstract}

DOI: $10.1103 /$ PhysRevC.96.054312

\section{INTRODUCTION}

Over the past decade, nuclear structure for mediummass nuclei have been extensively investigated by $a b$ inito many-body approaches such as the coupled-cluster method (CCM) [1-9], in-medium similarity renormalization group (IM-SRG) approach [10-12], and self-consistent Green's function method [13-15]. For instance, the reproduction of ground-state energies for oxygen isotopes is one of the most successful examples with such methods $[5,11,14]$. This finding supports the importance of the contributions from three-body forces for exotic nuclear structure. Also, the CCM casts light on the size of atomic nucleus from the study of neutron and weak-charge distributions of the ${ }^{48} \mathrm{Ca}$ nucleus [16]. In addition, the IM-SRG stimulates discussion on nuclear shape deformations with such ab initio approaches [17], which follows the extension of the method to solve the valence-space problem for open-shell nuclei $[18,19]$.

Similar to these methods, the unitary-model-operator approach (UMOA) [20-22] is also applicable to the investigation for the medium-mass nuclei. The UMOA was first introduced by Providência and Shakin to study the effects of short-range correlations on nuclear wave functions [23,24]. In those studies, the correlation operator was empirically determined. After these exploratory studies, the determination of correlation operators has been established based on a Hermitian effective-interaction theory [25,26]. Applying this to many-body systems, the Okubo-Lee-Suzuki transformation is done for many-body Hamiltonians to decouple the $n$-particle $n$-hole ( $n \mathrm{p} n \mathrm{~h}$ ) excitations. In other words, the UMOA is natural extension of the Hartree-Fock (HF) method where only the $1 \mathrm{p} 1 \mathrm{~h}$ decoupling is considered [27]. For actual applications of the UMOA, only the $2 \mathrm{p} 2 \mathrm{~h}$ excitations have been decoupled with the $0 \mathrm{p} 0 \mathrm{~h}$ state. As mentioned in our previous publications
[28,29], the results strongly depend on the harmonic-oscillator energy $\hbar \omega$, even if these are converged with respect to the size of model space. It can be an issue to obtain reliable converged results which should be free from the underlying parameters (the model-space sizes and $\hbar \omega$ values) and also to compare with the other $a b$ initio results.

According to the recent CCM study [30], the $\hbar \omega$ dependence can be drastically reduced by considering the one-body correlations. In the present work, we follow this study and introduce the one-body correlation operator in the UMOA in addition to the two-body correlation operator included already in the former calculations. We expect that the one-body correlation operator optimizes single-particle states and controls the $\hbar \omega$ dependence on calculated observables. The main purpose of this work is to demonstrate how the introduction of the one-body correlation operator in the UMOA works well and to discuss the $\hbar \omega$ dependence of the ground-state energy and point-nucleon radius for the ${ }^{4} \mathrm{He}$ nucleus taken as a test case.

The outline of this paper is as follows. In Sec. II, we represent the theoretical framework of the UMOA focusing on the difference between the previous and current calculation procedures. Then, we show the results of ground-state energies and point-nucleon radii for ${ }^{4} \mathrm{He}$ to check how the $\hbar \omega$ dependence on these observables can be removed by the implementation of the one-body correlation operator in Sec. III. We also compare the UMOA results with the other ab initio ones with the same interactions. Finally, we summarize the present work in Sec. IV.

\section{UNITARY-MODEL-OPERATOR APPROACH}

Before discussing how the $1 \mathrm{p} 1 \mathrm{~h}$ decoupling works well in the UMOA, we present theoretical structure in this section. In 
Sec. II A, general transformed Hamiltonians in the UMOA are introduced to help the understanding of the difference between the formulations employed in the earlier and this studies. Then, how to truncate the model space in the UMOA is discussed in Sec. II B. Section IIC describes the actual calculation procedure, focusing on the difference between the formulations employed in the former studies and this one. Finally, in Sec. IID, we mention how to calculate the observables in the UMOA.

\section{A. Similarity transformation}

We begin with the many-body Schrödinger equation,

$$
H|\Psi\rangle=E_{\text {g.s. }}|\Psi\rangle,
$$

with the ground-state energy eigenvalue $E_{\text {g.s. }}$ and eigenvector $|\Psi\rangle$. Here, the operator $H$ is a general intrinsic Hamiltonian,

$$
H=\sum_{i}^{A} \frac{\mathbf{p}_{i}^{2}}{2 m}-T_{\text {c.m. }}+\sum_{i<j}^{A} V_{i j}+\sum_{i<j<k}^{A} V_{i j k}+\cdots
$$

with the mass number $A$, the momentum of the $i$ th nucleon $\mathbf{p}_{i}$, the nucleon mass $m$, the $N N$ interaction $V_{i j}$, and the threenucleon interaction $V_{i j k}$. The center-of-mass kinetic energy $T_{\text {c.m. }}$ can be described with the one- and two-body terms as $T_{\text {c.m. }}=\sum_{i}^{A} \frac{\mathbf{p}_{i}^{2}}{2 A m}+\sum_{i<j}^{A} \frac{\mathbf{p}_{i} \cdot \mathbf{p}_{j}}{A m}$. Then, the Hamiltonian can be rewritten as

$$
H=\sum_{i}^{A} t_{i}+\sum_{i<j}^{A} v_{i j}+\sum_{i<j<k}^{A} v_{i j k}+\cdots
$$

with the one-, two-, and three-body terms, $t_{i}=\frac{A-1}{A} \frac{\mathbf{p}_{i}^{2}}{2 m}, v_{i j}=$ $V_{i j}-\mathbf{p}_{i} \cdot \mathbf{p}_{j} / A m$, and $v_{i j k}=V_{i j k}$, respectively.

To decouple the $0 \mathrm{p} 0 \mathrm{~h}$ state with $n \mathrm{p} n \mathrm{~h}$ states, the similarity transformation of the original Hamiltonian can be done as

$$
\widetilde{H}=U^{\dagger}(H+W) U-U^{\dagger} W U
$$

with the unitary operator $U$ and the auxiliary potential $W$. Here, the auxiliary potential is introduced as

$$
W=\sum_{i}^{A} w_{i}+\sum_{i<j}^{A} w_{i j}+\sum_{i<j<k}^{A} w_{i j k}+\cdots
$$

so as to take into account the in-medium effects. The operator $w_{i_{1} \ldots i_{n}}$ is the $n$-body auxiliary potential. So far, the auxiliary potential $w_{i_{1} \cdots i_{n}}$ can be taken arbitrarily but is determined selfconsistently as discussed in the end of Sec. II A. With the transformation (4), the original Schrödinger equation, Eq. (1), is also transformed to

$$
\widetilde{H}|\Phi\rangle=E|\Phi\rangle
$$

with the reference state $|\Phi\rangle$ multiplied by the unitary operator $U$ as

$$
|\Phi\rangle=U^{\dagger}|\Psi\rangle .
$$

Since the reference state is arbitrary in principle, we take $|\Phi\rangle$ as a single Slater determinant such as the particle-hole vacuum. In the UMOA, the unitary-transformation operator $U$ is defined as the product of exponential operators up to the $A$-body terms [27],

$$
U=e^{S^{(1)}} e^{S^{(2)}} \cdots e^{S^{(A)}} .
$$

The exponents $S^{(1)}, S^{(2)}, \ldots, S^{(n)}$ are the one-, two-,$\ldots$, and $n$-body correlation operators. They are defined as

$$
\begin{aligned}
S^{(1)} & =\sum_{i}^{A} s_{i}, \\
S^{(2)} & =\sum_{i<j}^{A} s_{i j}, \\
S^{(n)} & =\sum_{i_{1}<\cdots<i_{n}}^{A} s_{i_{1} \cdots i_{n}},
\end{aligned}
$$

respectively. Here, $s_{i_{1} \cdots i_{n}}$ is the correlation operator acting on $n$ particles labeled by $i_{1}, \ldots, i_{n}$. The correlation operators $S^{(n)}$ are anti-Hermitian and satisfy

$$
S^{(n) \dagger}=-S^{(n)},
$$

so that the transformation operator $U$ is unitary.

Generally, the transformed Hamiltonian $\widetilde{H}$ is expanded by the Baker-Campbell-Hausdorff $(\mathrm{BCH})$ formula as found in the CCM [8]. It is because the $\mathrm{BCH}$ expansion terminates with the finite order and is actually one of the advantages in the $\mathrm{CCM}$. In contrast, the $\mathrm{BCH}$ expansion does not terminate with the finite order in the UMOA, as the correlation operators do not commute with each other. Therefore, the UMOA employs another type of expansion known as the cluster expansion [23]. Following the cluster expansion, we decompose $\widetilde{H}$ into

$$
\widetilde{H}=\widetilde{H}^{(1)}+\widetilde{H}^{(2)}+\widetilde{H}^{(3)}+\cdots,
$$

according to the number of interacting particles. Note that the three- and higher-body terms can be induced by the transformation, even if the initial Hamiltonian includes up to the two-body interaction. For clarification, we show the explicit expressions of the one-, two-, and $n$-body cluster terms:

$$
\begin{aligned}
& \widetilde{H}^{(1)}=\sum_{i}^{A} \widetilde{h}_{i}, \\
& \widetilde{H}^{(2)}=\sum_{i<j}^{A} \widetilde{v}_{i j}-\sum_{i}^{A} \widetilde{w}_{i}, \\
& \widetilde{H}^{(n)}=\sum_{i_{1}<\cdots<i_{n}}^{A} \widetilde{v}_{i_{1} \cdots i_{n}}-\sum_{i_{1}<\cdots<i_{n-1}}^{A} \widetilde{w}_{i_{1} \cdots i_{n-1}} \\
& \text { for } \quad 3 \leqslant n \leqslant A
\end{aligned}
$$


with the terms introduced as

$$
\begin{aligned}
\tilde{h}_{1}= & e^{-s_{1}} h_{1} e^{s_{1}}=e^{-s_{1}}\left(t_{1}+w_{1}\right) e^{s_{1}} \\
\widetilde{v}_{12}= & e^{-s_{12}} e^{-\left(s_{1}+s_{2}\right)}\left(h_{1}+h_{2}+v_{12}+w_{12}\right) e^{s_{1}+s_{2}} e^{s_{12}}-\left(\widetilde{h}_{1}+\widetilde{h}_{2}\right), \\
\widetilde{v}_{i_{1} \ldots i_{n}}= & e^{-s_{1} \ldots n} \ldots e^{-\left(\sum_{i<j} s_{i j}\right)} e^{-\left(\sum_{i} s_{i}\right)}\left(\sum_{i}^{n} h_{i}+\sum_{k=2}^{n} \sum_{i<\cdots<i_{k}}^{n} v_{i_{1} \cdots i_{k}}+\sum_{k=2}^{n} \sum_{i_{1}<\cdots<i_{k}}^{n} w_{i_{1} \cdots i_{k}}\right) e^{\sum_{i} s_{i}} e^{\sum_{i<j} s_{i j}} \ldots e^{s_{1 \ldots n}} \\
& -\left(\sum_{i}^{n} \widetilde{h}_{i}+\sum_{k=2}^{n-1} \sum_{i_{1}<\cdots<i_{k}}^{n} \widetilde{v}_{i_{1} \cdots i_{k}}\right), \text { for } 3 \leqslant n \leqslant A .
\end{aligned}
$$

The transformed auxiliary potentials $\widetilde{w}_{1}, \widetilde{w}_{12}, \ldots$ in Eqs. (14)(16) are, in principle, arbitrary, but the determinations of them are crucial in the actual calculation. In order to determine these transformed auxiliary potentials, we recall the one-body potential appeared in the HF method. In the HF calculations, the one-body potential cancels with the bubble-diagram contributions of the two-body interaction. This procedure is applied directly to the UMOA. Since the transformed Hamiltonian contains many-body transformed interactions and auxiliary potentials, the bubble-diagram contributions come from $\tilde{v}_{12}$, $\widetilde{v}_{123}, \ldots$ and $\widetilde{w}_{1}, \widetilde{w}_{12}, \ldots$ The conditions of the cancellation can be represented diagrammatically in Fig. 1. The analytical expressions corresponding to Fig. 1 are

$$
\begin{gathered}
\sum_{\lambda \leqslant \rho_{F}}\left\langle\alpha \lambda\left|\widetilde{v}_{12}\right| \beta \lambda\right\rangle+\frac{1}{2 !} \sum_{\lambda \mu \leqslant \rho_{F}}\left\langle\alpha \lambda \mu\left|\widetilde{v}_{123}\right| \beta \lambda \mu\right\rangle+\cdots \\
\quad-\left\langle\alpha\left|\widetilde{w}_{1}\right| \beta\right\rangle-\sum_{\lambda \leqslant \rho_{F}}\left\langle\alpha \lambda\left|\widetilde{w}_{12}\right| \beta \lambda\right\rangle-\cdots=0
\end{gathered}
$$

for the one-body term and

$$
\begin{aligned}
& \sum_{\lambda \leqslant \rho_{F}}\left\langle\alpha \beta \lambda\left|\widetilde{v}_{123}\right| \gamma \delta \lambda\right\rangle+\frac{1}{2 !} \sum_{\lambda \mu \leqslant \rho_{F}}\left\langle\alpha \beta \lambda \mu\left|\widetilde{v}_{1234}\right| \gamma \delta \lambda \mu\right\rangle+\cdots \\
& \quad-\left\langle\alpha \beta\left|\widetilde{w}_{12}\right| \gamma \delta\right\rangle-\sum_{\lambda \leqslant \rho_{F}}\left\langle\alpha \beta \lambda\left|\widetilde{w}_{123}\right| \gamma \delta \lambda\right\rangle-\cdots=0
\end{aligned}
$$

for the two-body term. Here, $\rho_{F}$ denotes the Fermi level and $\left|\alpha_{1} \ldots \alpha_{n}\right\rangle$ is antisymmetrized and normalized $n$-body state. The conditions of the cancellation for three- and higher body

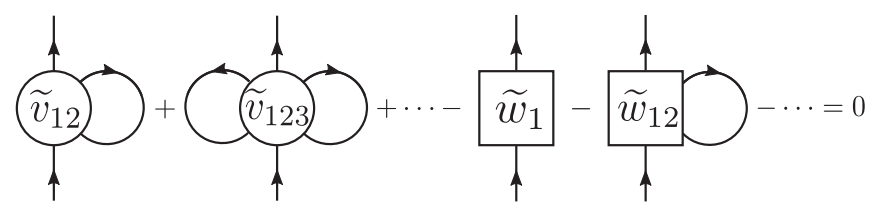

(a)

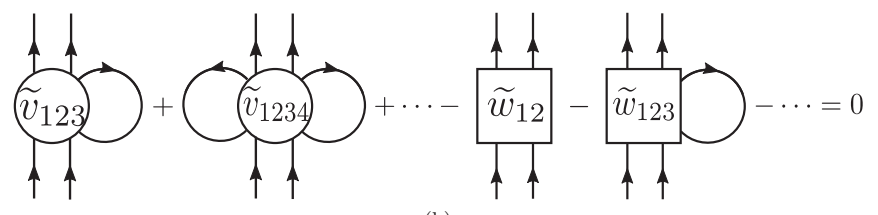

(b)

FIG. 1. Cancellations of bubble-diagram contributions for the one-body (a) and two-body (b) parts. terms are given in the same way. Thus, the matrix elements of the transformed auxiliary potentials are [27,31]

$$
\begin{aligned}
\left\langle\alpha\left|\widetilde{w}_{1}\right| \beta\right\rangle= & \sum_{\lambda_{1} \leqslant \rho_{F}}\left\langle\alpha \lambda_{1}\left|\widetilde{v}_{12}\right| \beta \lambda_{1}\right\rangle \\
& -\frac{1}{2 !} \sum_{\lambda_{1} \lambda_{2} \leqslant \rho_{F}}\left\langle\alpha \lambda_{1} \lambda_{2}\left|\widetilde{v}_{123}\right| \beta \lambda_{1} \lambda_{2}\right\rangle+\cdots
\end{aligned}
$$

for the one-body potential, and

$$
\begin{aligned}
\left\langle\alpha \beta\left|\tilde{w}_{12}\right| \gamma \delta\right\rangle= & \sum_{\lambda_{1} \leqslant \rho_{F}}\left\langle\alpha \beta \lambda_{1}\left|\widetilde{v}_{123}\right| \gamma \delta \lambda_{1}\right\rangle \\
& -\frac{1}{2 !} \sum_{\lambda_{1} \lambda_{2} \leqslant \rho_{F}}\left\langle\alpha \beta \lambda_{1} \lambda_{2}\left|\widetilde{v}_{1234}\right| \gamma \delta \lambda_{1} \lambda_{2}\right\rangle+\cdots
\end{aligned}
$$

for the two-body potential.

Furthermore, the auxiliary potentials $w_{1}, w_{12}, \ldots$ in Eqs. (17)-(19) are related to the transformed auxiliary potentials $\widetilde{w}_{1}, \widetilde{w}_{12}, \ldots$ through the relevant inverse transformations $[27,31]$,

$$
\begin{aligned}
w_{1} & =e^{s_{1}} \widetilde{w}_{1} e^{-s_{1}}, \\
w_{12} & =e^{s_{1}+s_{2}} e^{s_{12}}\left(\widetilde{w}_{1}+\widetilde{w}_{2}+\widetilde{w}_{12}\right) e^{-s_{12}} e^{-\left(s_{1}+s_{2}\right)}-\left(w_{1}+w_{2}\right) .
\end{aligned}
$$

As found in Refs [27,30,31], this choice of the transformed auxiliary potentials gives the normal ordered $\widetilde{H}$ with respect to $|\Phi\rangle$.

The essential point in the UMOA is to determine the correlation operators. These are determined so that the transformed Hamiltonian does not induce the particle-hole excitations. There are a number of studies about the correlation operators (see, for example, Refs. [32-34]). For brevity, the determination of correlation operators is given in Appendix A. Once the correlation operators are determined, one can build up the transformed Hamiltonian with Eqs. (13)-(19).

\section{B. Model space}

In this subsection, we present the choice of the model space employed in earlier and current UMOA calculations. To obtain converged results in relatively smaller mode spaces, the two-step decoupling method has been applied in the earlier UMOA [21,22]. Figures 2(a) and 2(b) schematically show how 


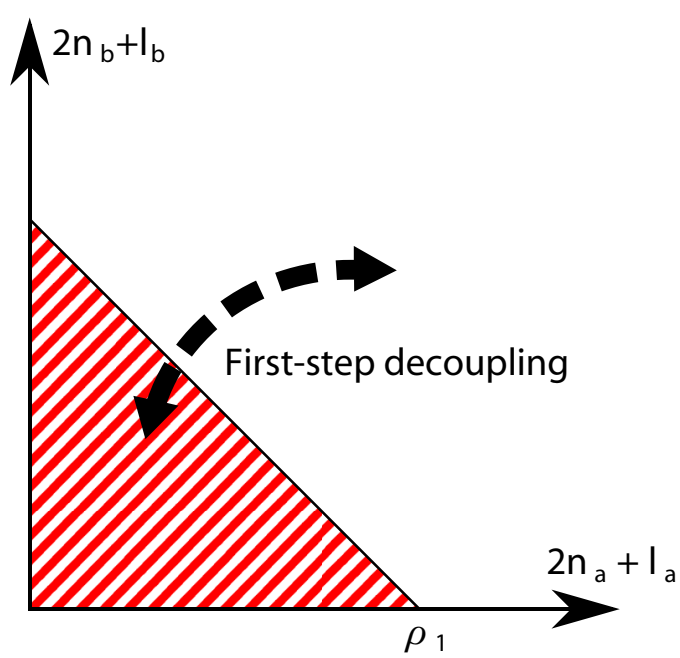

(a)

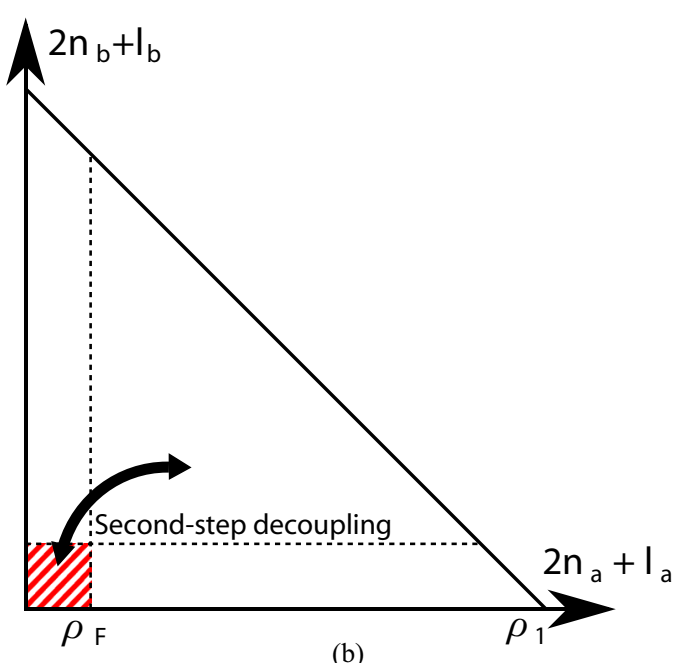

(b)

FIG. 2. Illustrations of the model space and its decoupling employed in the previous work. The first-step decoupling (a) denotes the decoupling between the outside and inside (shaded area) of our model space. The second-step decoupling (b) means the decoupling of $2 \mathrm{p} 2 \mathrm{~h}$ excitations with $0 \mathrm{p} 0 \mathrm{~h}$ state (shaded area).

to decouple the model space in the earlier calculations. In the first-step decoupling, the initial Hamiltonian is transformed so that the model space [the shaded area in Fig. 2(a)] and its complement are decoupled. The aim of this step is to make bare $N N$ interactions able to be handled without any explicit softening of input interactions. Since this decoupling is done in the huge space to take into account short-range correlations of input bare $N N$ interactions, the decoupling equation is solved with the relative and center-of-mass coordinates. Thus, our model space has to be truncated to be a triangle shape in the two-body space by $\rho_{1}=\max \left(2 n_{a}+l_{a}+2 n_{b}+l_{b}\right)$. Here, $n_{a}$ and $l_{a}$ are the nodal and azimuthal quantum numbers of the harmonic-oscillator (HO) basis state $a$, respectively. By employing this effective interaction through the first-step decoupling, we further decouple the $2 \mathrm{p} 2 \mathrm{~h}$ excitations with the reference state, which is illustrated by the solid arrow in Fig. 2(b). After the second-step decoupling, we construct the

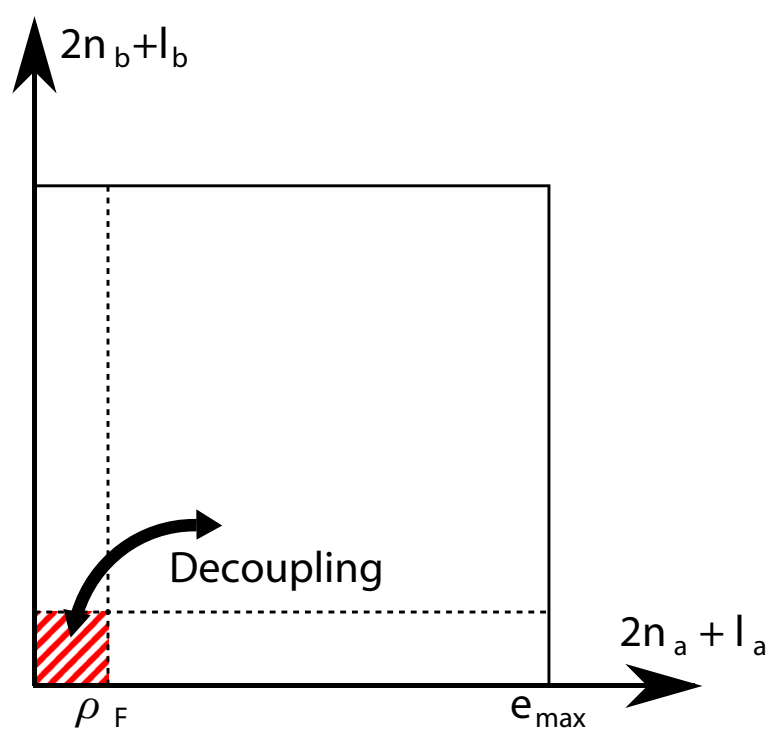

FIG. 3. Illustrations of the model space and its decoupling employed in the present work. The shaded area indicates our reference state. The arrow means the decoupling of $1 \mathrm{p} 1 \mathrm{~h}$ and $2 \mathrm{p} 2 \mathrm{~h}$ excitations with the reference state.

transformed Hamiltonian and obtain the observables using the transformed operators as discussed in Sec. II D.

In the first-step decoupling, the angle-average approximation is used for the Pauli exclusion operator. The investigation of this approximation, at least for finite nuclei, may not be sufficient and can cause uncontrollable uncertainties. As discussed in Refs. [35-37], it was reported that there is the non-negligible difference between the results with and without the angle-average approximation in the nuclear matter calculations. Since we would like to examine the validity of the UMOA without any uncontrollable approximations, we do not employ the first-step decoupling in the present work. Alternatively, we soften input bare interactions via low momentum or similarity renormalization group techniques. Therefore, we consider only the process for the decoupling of the $1 \mathrm{p} 1 \mathrm{~h}$ and $2 \mathrm{p} 2 \mathrm{~h}$ excitations on top of the reference state. Then, the choice of the model space is no longer restricted to the triangle shape. Here, we employ the simplest square model space defined by $e_{\max }=\max \left(2 n_{a}+l_{a}\right)=\max \left(2 n_{b}+l_{b}\right)$, as shown in Fig. 3. The decoupling of the $1 \mathrm{p} 1 \mathrm{~h}$ and $2 \mathrm{p} 2 \mathrm{~h}$ excitations is indicated by the solid arrow in Fig. 3 .

\section{Numerical implementation}

In this subsection, we discuss the numerical implementation of the UMOA with the 1p1h decoupling examined in the present work. In this study, we treat only two-body interactions and do not include any three- and higher many-body ones in the initial Hamiltonian, Eqs. (2) and (3). In addition, we keep only the one- and two-body cluster terms and correlation operators. Thus, two- and many-body auxiliary potentials in Eq. (5) are dropped out and only one-body auxiliary potentials $W=\sum_{i} w_{i}$ are left. Under these conditions, the flow chart of the actual computation is illustrated schematically in Fig. 4. Each step of the procedure is also listed as follows: 


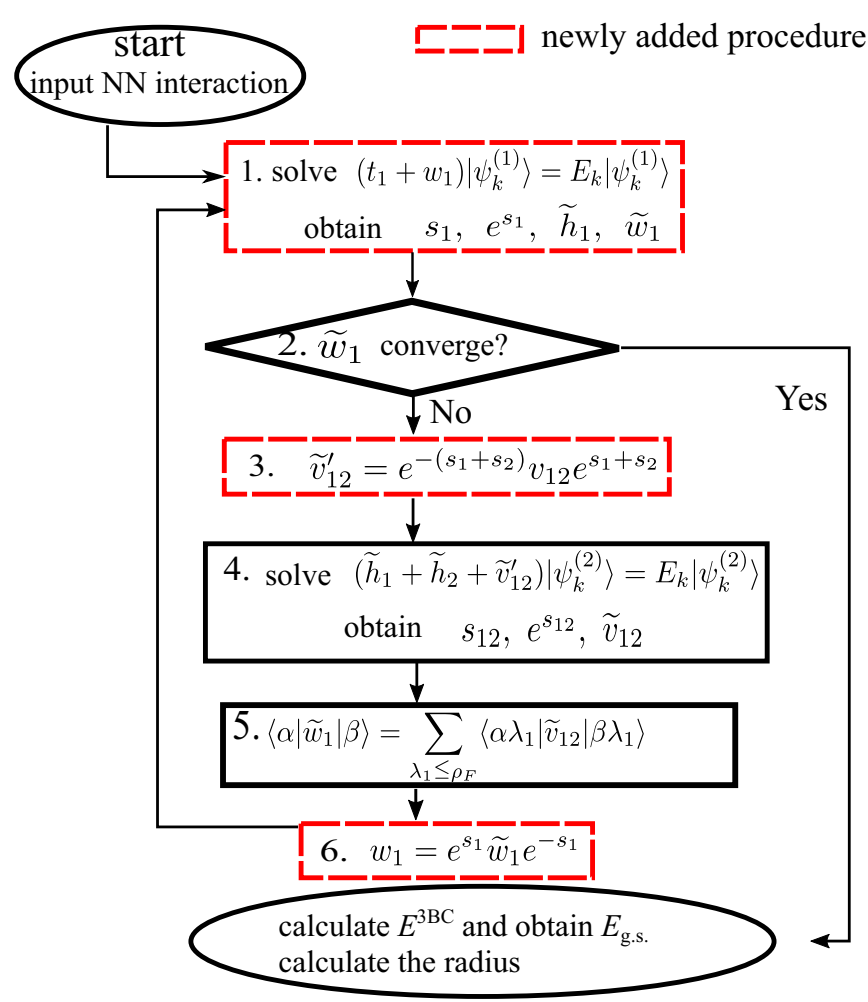

FIG. 4. Flow chart of the actual calculation. The steps surrounded with the dashed line $(1,3$, and 6$)$ are related to the procedure on the $1 \mathrm{p} 1 \mathrm{~h}$ decoupling newly added this time. See text for details.

(1) The one-body Schrödinger equation,

$$
\left(t_{1}+w_{1}\right)\left|\psi_{k}^{(1)}\right\rangle=E_{k}\left|\psi_{k}^{(1)}\right\rangle
$$

is solved and $s_{1}, e^{s_{1}}, \widetilde{h}_{1}, \widetilde{t}_{1}$, and $\widetilde{w}_{1}$ are obtained by using Eqs. (A6), (A10), and (17), respectively.

(2) The calculation is performed iteratively until $\max \left(\left|\widetilde{w}_{1}^{\text {(new) }}-\widetilde{w}_{1}^{\text {(old) }}\right|\right)<10^{-5}$ is satisfied. Here, $\widetilde{w}_{1}^{\text {(new) }}$ and $\widetilde{w}_{1}^{\text {(old) }}$ are the one-body fields obtained at the current and previous iteration steps, respectively.

(3) By using the operator $e^{s_{1}}$ obtained in step 1, the original two-body interaction is transformed as

$$
\widetilde{v}_{12}^{\prime}=e^{-\left(s_{1}+s_{2}\right)} v_{12} e^{s_{1}+s_{2}} .
$$

(4) The two-body Schrödinger equation,

$$
\begin{aligned}
& \left(P^{(2)}+Q^{(2)}\right)\left(\tilde{h}_{1}+\widetilde{h}_{2}+\widetilde{v}_{12}^{\prime}\right)\left(P^{(2)}+Q^{(2)}\right)\left|\psi_{k}^{(2)}\right\rangle \\
& \quad=E_{k}\left|\psi_{k}^{(2)}\right\rangle,
\end{aligned}
$$

is solved and $s_{12}, e^{s_{12}}$, and $\widetilde{v}_{12}$ are obtained by using Eqs. (A6), (A10), and (18), respectively.

(5) By taking the normal ordering with respect to the Fermi level,

$$
\left\langle\alpha\left|\widetilde{w}_{1}\right| \beta\right\rangle=\sum_{\lambda \leqslant \lambda_{F}}\left\langle\alpha \lambda\left|\widetilde{v}_{12}\right| \beta \lambda\right\rangle,
$$

we obtain new $\widetilde{w}_{1}$.

(6) Applying the inverse transformation,

$$
w_{1}=e^{s_{1}} \widetilde{w}_{1} e^{-s_{1}},
$$

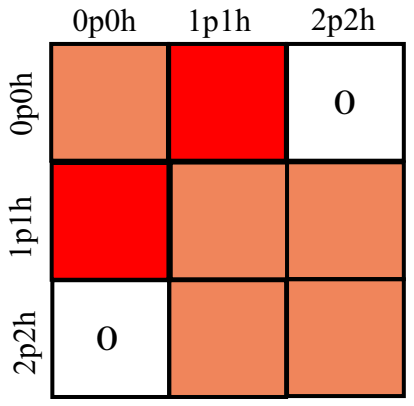

(a)

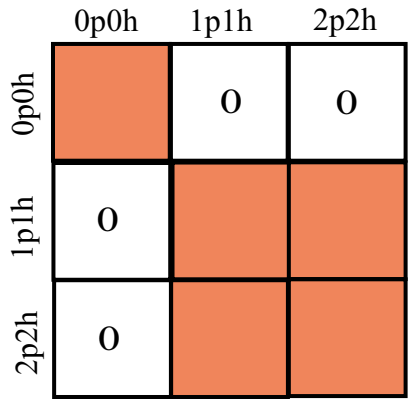

(b)
FIG. 5. Schematic representations of the transformed Hamiltonians without [left; panel (a)] and with [right; panel (b)] the one-body correlation operator.

we get $w_{1}$ to be substituted into the one-body Schrödinger equation in step 1.

After the calculation procedure described above, the correlation operators $s_{1}$ and $s_{12}$ are evaluated. Using $s_{1}$ and $s_{12}$, we can construct the transformed Hamiltonian and then obtain the ground-state energy and wave function. Moreover, we can obtain the other expectation values of observables with the same transformed operators as discussed in Sec. II D. This is one of the advantages about effective operators in the UMOA.

\section{Ground-state energy and radius}

Here, we present how to calculate the ground-state energy and root-mean-square radius in the UMOA. After the transformation discussed in Sec. II C, we construct the transformed Hamiltonian up to the two-body parts with Eqs. (14), (15), (17), (18), and (29). Figure 5 shows the schematic representations of the transformed Hamiltonians with and without the one-body correlation operator. In previous publications [28,29], we have employed the Hamiltonian without the $1 \mathrm{p} 1 \mathrm{~h}$ decoupling $\left(S^{(1)}=0\right)$ as shown schematically in Fig. 5(a) to evaluate the ground-state energy and radius. Instead, we diagonalized the transformed Hamiltonian in the $0 \mathrm{p} 0 \mathrm{~h}$ and $1 \mathrm{p} 1 \mathrm{~h}$ space to take into account the effect of off-diagonal components between the $0 \mathrm{p} 0 \mathrm{~h}$ and $1 \mathrm{p} 1 \mathrm{~h}$ states. In the present formulation, the matrix elements between the $0 \mathrm{p} 0 \mathrm{~h}$ and $1 \mathrm{p} 1 \mathrm{~h}$ states vanish from the beginning, because of the $1 \mathrm{p} 1 \mathrm{~h}$ decoupling by the one-body correlation operator $S^{(1)}$ as shown in Fig. 5(b). The ground-state energy is dictated with the transformed Hamiltonian and reference state as

$$
E_{\text {g.s. }}=\langle\Psi|H| \Psi\rangle=(\langle\Psi| U) U^{\dagger} H U\left(U^{\dagger}|\Psi\rangle\right)=\langle\Phi|\widetilde{H}| \Phi\rangle .
$$

The rightmost term in Eq. (31) corresponds to the zero-body term after taking the normal ordering with respect to the reference state $|\Phi\rangle$. It is approximated up to the third order 
of expansion as

$$
\begin{aligned}
E_{\text {g.s. }} \approx & \sum_{\lambda \leqslant \rho_{F}}\left\langle\lambda\left|\widetilde{h}_{1}\right| \lambda\right\rangle+\frac{1}{2 !} \sum_{\lambda \mu \leqslant \rho_{F}}\left\langle\lambda \mu\left|\widetilde{v}_{12}\right| \lambda \mu\right\rangle \\
& -\sum_{\lambda \leqslant \rho_{F}}\left\langle\lambda\left|\widetilde{w}_{1}\right| \lambda\right\rangle+E^{3 \mathrm{BC}} \\
= & \sum_{\lambda \leqslant \rho_{F}}\left\langle\lambda\left|\widetilde{t}_{1}\right| \lambda\right\rangle+\frac{1}{2 !} \sum_{\lambda \mu \leqslant \rho_{F}}\left\langle\lambda \mu\left|\widetilde{v}_{12}\right| \lambda \mu\right\rangle+E^{3 \mathrm{BC}},
\end{aligned}
$$

where $\widetilde{t}_{1}\left(=e^{-s_{1}} t_{1} e^{s_{1}}\right)$ is the one-body transformed kinetic energy term. The three-body cluster term $E^{3 \mathrm{BC}}$ is evaluated a posteriori as

$$
E^{3 \mathrm{BC}}=\frac{1}{3 !} \sum_{\lambda \mu \nu \leqslant \rho_{F}}\left\langle\lambda \mu \nu\left|\widetilde{v}_{123}\right| \lambda \mu \nu\right\rangle,
$$

which is taken into account through order $S^{(2) 2}$ [20]. From our experience, this approximation is rather good. The convergence with respect to the cluster expansion will be discussed in Sec. III.

One of the advantages in the UMOA is the usage of the same transformed operators for energies to obtain the other observables. Although, in principle, one can also use the transformed operator in the CCM, one has to solve the coupled-cluster equation for both left and right eigenvectors to construct the transformation operator due to the nonHermiticity. In practical applications, the Hellmann-Feynman theorem is often applied to obtain the observables other than energies, as found in recent studies (see, e.g., Ref. [8]). On the other hand, the calculation of observables is rather simple in the UMOA. For an explicit demonstration, we show how to calculate the radius in the following. Let $r^{2}$ be the squared point-nucleon radius operator defined as

$$
r^{2}=\frac{1}{A} \sum_{i}\left(\mathbf{r}_{i}-\mathbf{R}_{\mathrm{c} . \mathrm{m} .}\right)^{2}
$$

with the coordinate vector of $i$ th nucleon $\mathbf{r}_{i}$ and of the center of mass $\mathbf{R}_{\text {c.m. }}$. Similar to the evaluation of ground-state energy, the squared radius operator $r^{2}$ is decomposed into one- and two-body operators,

$$
r^{2}=r^{2(1)}+r^{2(2)},
$$

where $r^{2(1)}$ and $r^{2(2)}$ are the one- and two-body parts, respectively, as

$$
\begin{aligned}
& r^{2(1)}=\frac{1}{A}\left(1-\frac{1}{A}\right) \sum_{i=1}^{A} \mathbf{r}_{i}^{2}=\sum_{i=1}^{A} r_{i}^{2}, \\
& r^{2(2)}=-2 \frac{1}{A^{2}} \sum_{i<j}^{A} \mathbf{r}_{i} \cdot \mathbf{r}_{j}=\sum_{i<j}^{A} r_{i j}^{2} .
\end{aligned}
$$

With the aid of the unitary operator $U$, the expectation value can be expressed by the transformed operator $\widetilde{r}^{2}$ and the reference state $|\Phi\rangle$ as

$$
\left\langle\Psi\left|r^{2}\right| \Psi\right\rangle=\left\langle\Phi\left|\widetilde{r}^{2}\right| \Phi\right\rangle
$$

with the transformed radius operator $\widetilde{r}^{2}$

$$
\tilde{r}^{2}=U^{\dagger} r^{2} U
$$

Then, we carry out the cluster expansion of the transformed operator $\widetilde{r}^{2}$,

$$
\widetilde{r}^{2}=\widetilde{r}^{2(1)}+\widetilde{r}^{2(2)}+\cdots .
$$

Here, the one- and two-body cluster terms are generated as

$$
\widetilde{r}^{2(1)}=\sum_{i} \widetilde{r}_{i}^{2}, \quad \widetilde{r}^{2(2)}=\sum_{i<j} \widetilde{r}_{i j}^{2},
$$

with

$$
\begin{aligned}
& \widetilde{r}_{1}^{2}=e^{-s_{1}} r_{1}^{2} e^{s_{1}}, \\
& \widetilde{r}_{12}^{2}=e^{-s_{12}} e^{-\left(s_{1}+s_{2}\right)}\left(r_{1}^{2}+r_{2}^{2}+r_{12}^{2}\right) e^{s_{1}+s_{2}} e^{s_{12}}-\left(\widetilde{r}_{1}^{2}+\widetilde{r}_{2}^{2}\right) .
\end{aligned}
$$

The expectation value is equal to the normal-ordered zero-body term,

$$
\begin{aligned}
\left\langle\Phi\left|\widetilde{r}^{2}\right| \Phi\right\rangle & =\sum_{\lambda \leqslant \rho_{F}}\left\langle\lambda\left|\widetilde{r}^{2(1)}\right| \lambda\right\rangle+\frac{1}{2} \sum_{\lambda \mu \leqslant \rho_{F}}\left\langle\lambda \mu\left|\widetilde{r}^{2(2)}\right| \lambda \mu\right\rangle+\cdots \\
& \approx \widetilde{r}^{2(1 \mathrm{BC})}+\widetilde{r}^{2(2 \mathrm{BC})} .
\end{aligned}
$$

Here, $\widetilde{r}^{2(1 \mathrm{BC})}$ and $\widetilde{r}^{2(2 \mathrm{BC})}$ are the contributions of one- and two-body transformed radius operators, respectively. In actual calculations, we truncate the cluster expansion up to the second order to evaluate the radius as in Eq. (45).

\section{RESULTS AND DISCUSSION}

In the earlier UMOA studies, the ground-state properties of ${ }^{16} \mathrm{O},{ }^{40} \mathrm{Ca}$, and ${ }^{56} \mathrm{Ni}$ have been mainly discussed with realistic $N N$ interactions [20-22,28,29]. Only the decoupling of the $2 \mathrm{p} 2 \mathrm{~h}$ excitations has been considered, i.e., $U=e^{S^{(2)}}$ in Eq. (8). The calculated results, especially for radii, strongly depend on the $\hbar \omega$ values and are difficult to judge the reliability from $a b$ initio point of view. Following the success to reduce the $\hbar \omega$ dependence in the CCM [30], we naturally extend the formalism and introduce the one-body correlation operator $S^{(1)}$ to the UMOA in the present study. As the validation of the effectiveness, we show the numerical results of ground-state energy and point-nucleon radius of ${ }^{4} \mathrm{He}$ with the transformation $U=e^{S^{(1)}} e^{S^{(2)}}$. Then, we discuss the role of the one-body correlation operator $S^{(1)}$ in the UMOA to some extent.

The choice of the initial Hamiltonian for numerical calculations is one of the important issues. Nowadays, sophisticated nucleon-nucleon $(N N)$ interactions have been developed which reproduce the $N N$ scattering phase shift data with high precision, as well as the deuteron properties, such as the AV18 [38], CD-Bonn [39], and chiral EFT N ${ }^{3}$ LO interactions [40]. It is, however, difficult to apply directly such bare $N N$ interactions to our calculations, because of the strong coupling between low- and high-momentum regions. To get rid of this computational difficulty, we have applied to the earlier UMOA the effective interactions derived with some approximations which hamper reliable estimations of the uncertainty on calculated results (see, e.g., Refs. [20,21]). 
In the present work, we are interested in confirming the applicability of the UMOA through comparison to other $a b$ initio results. It is preferable to reduce the uncertainties coming from the effective interactions employed in the earlier UMOA as much as possible. Therefore, we omit such process in the earlier UMOA by using sophisticated softened interactions. For this purpose, we mainly use two types of $N N$ interactions. One is the low-momentum interaction $V_{\text {low k }}$ derived from the AV18 NN interaction [38] with the sharp cutoff $\Lambda=1.9 \mathrm{fm}^{-1}$. The other is the SRG-transformed chiral EFT $\mathrm{N}^{3} \mathrm{LO} N N$ interaction [40] with the cutoff $\lambda_{\mathrm{SRG}}=2.0 \mathrm{fm}^{-1}$ to compare with the recent $a b$ initio calculation results [2,41,42]. As the qualitative aspect between these two interactions is similar enough to discuss the role of $S^{(1)}$ in the UMOA, we mainly show the results with the low-momentum interaction $V_{\text {low k }}$ in the following discussion. Note that our results are not comparable directly to the experimental data due to the missing genuine three-body and induced many-body interactions which cannot be treated in the $V_{\text {low k }}$ formalism. We can, however, compare our present UMOA results with the other $a b$ initio ones obtained by using the same $N N$ interactions.

\section{A. Role of the one-body correlation operator}

Before discussing the importance of one-body correlations in the UMOA, Fig. 6 summarizes the dependence of the model-space size on the energy and point-nucleon radius of the ${ }^{4} \mathrm{He}$ ground state in the former and current UMOA. In the left panels, one-body correlations are approximately taken into account through the diagonalization in the $0 \mathrm{p} 0 \mathrm{~h}$ and $1 \mathrm{p} 1 \mathrm{~h}$ space as done in earlier works $[21,22,28,29]$. In the right panels, one-body correlations are explicitly included by the one-body correlation operator as in the present formalism. As seen in the figure, the convergence with respect to the size of the model space can be almost achieved around $e_{\max }=12$ both in the earlier (left panels) and current (right panels) formulations. Interestingly, in the earlier formalism (shown in the left panels), we can observe the artificial $\hbar \omega$ dependence even after the convergence for the model-space size is achieved. It is our motivation behind the employment of the one-body correlation operator in the UMOA to eliminate this artificial dependence. On the other hand, in the current formalism (shown in the right panels), the results show typical behavior for the $\hbar \omega$ and $e_{\max }$ parameters found in usual ab initio calculations; i.e., the $\hbar \omega$ dependence reduces as the model-space size increases. Now we can obtain the converged results by the UMOA calculations with sufficiently large model space, unlike before. In the figure, the Faddeev-Yakubovsky (FY) [41] and coupled-cluster single and double (CCSD) [2] results for the ground-state energy with the same $N N$ interaction are also shown for comparison.

After confirming the convergence of the results with respect to the parameters, $\hbar \omega$ and $e_{\max }$, Fig. 7 compares the ground-state energies (upper panel) and point-nucleon root-mean-square radii (lower panel) of ${ }^{4} \mathrm{He}$ as functions of $\hbar \omega$ in the earlier and current UMOA formalisms with and without the one-body correlation operator $S^{(1)}$. Note that we show the results only in $e_{\max }=12$, because the calculated results almost converge at $e_{\max }=12$ as found in Fig. 6. For example, the difference between our results for energies at $e_{\max }=12$ and $e_{\max }=14$ is order of $10 \mathrm{keV}$. It is sufficient for the present purpose of discussion. In the figure, the results without the one-body correlation operator $S^{(1)}$ (blue triangles) are almost parallel with the $\mathrm{HO} 0 \mathrm{p} 0 \mathrm{~h}$ results (black dashed line) both for the ground-state energies and point-nucleon radii. It implies that the operator $S^{(2)}$ does not change the trend of the $\hbar \omega$ dependence originating from the $\mathrm{HO} 0 \mathrm{pOh}$ reference state.
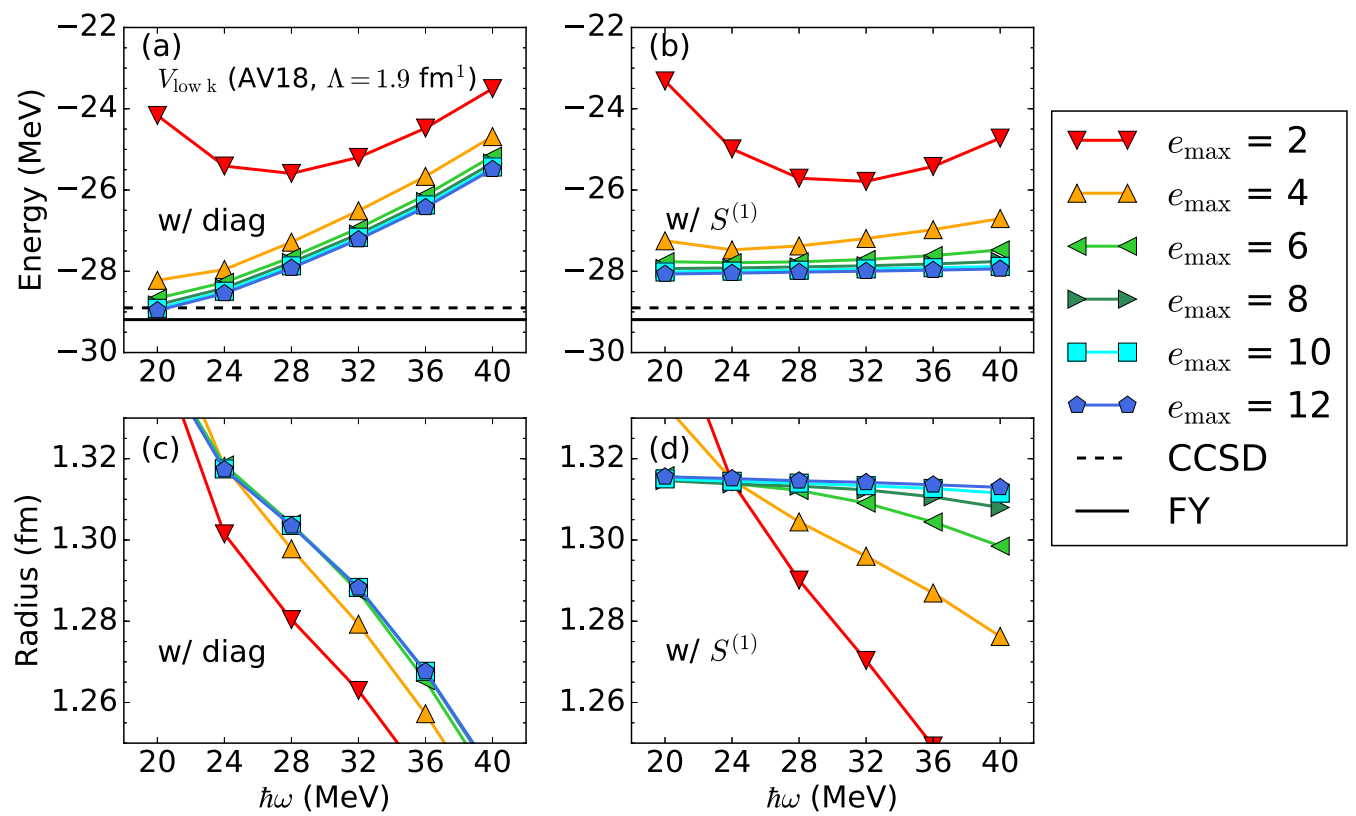

FIG. 6. Ground-state energies [upper panels (a) and (b)] and point-nucleon root-mean-square matter radii [lower panels (b) and (d)] of the ${ }^{4} \mathrm{He}$ nucleus as a functions of the HO energy $\hbar \omega$. In panels (a) and (c), one-body correlations are approximately taken into account through the diagonalization in $0 \mathrm{p} 0 \mathrm{~h}$ and $1 \mathrm{p} 1 \mathrm{~h}$ space as done in earlier works [21,22,28,29]. In panels (b) and (d), one-body correlations are explicitly included. Note that the results from "FY" (solid line) and "CCSD" are taken from Refs. [41] and [2], respectively. 

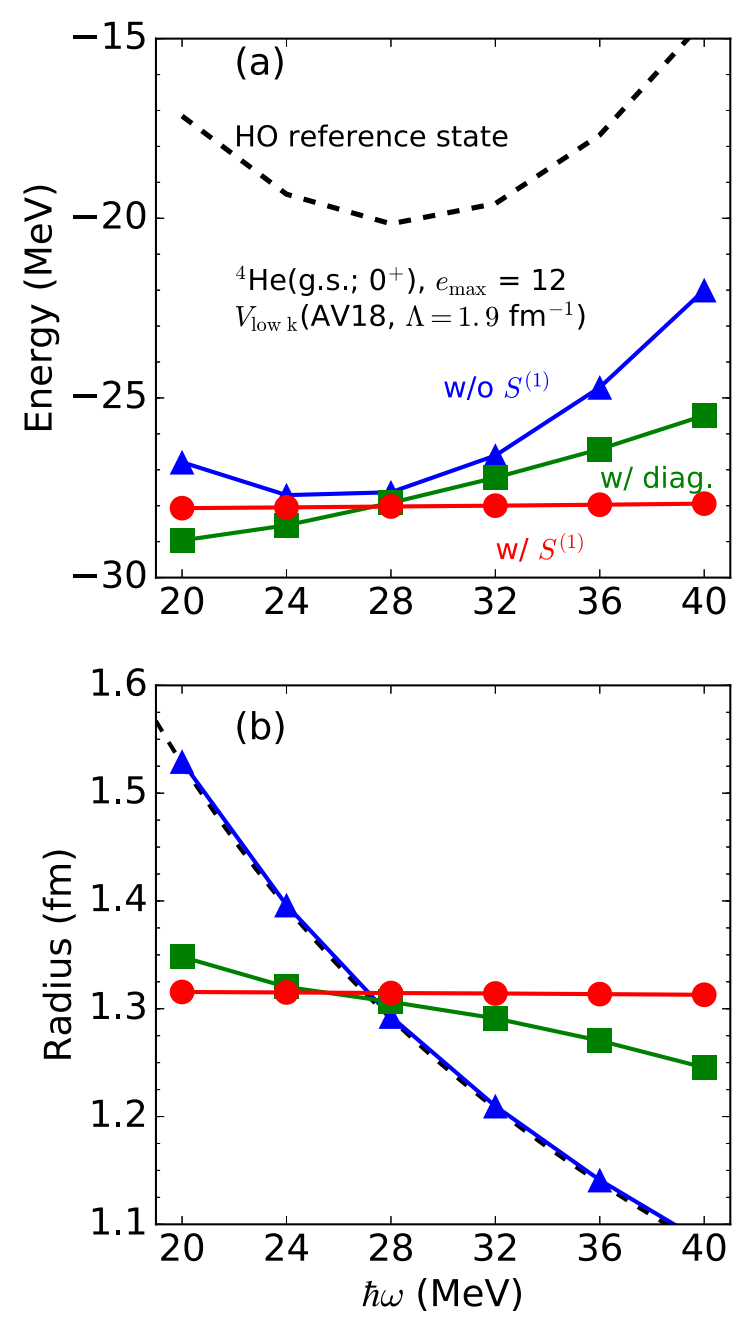

FIG. 7. Ground-state energies (upper panel) and point-nucleon root-mean-square matter radii (lower panel) of the ${ }^{4} \mathrm{He}$ nucleus as a functions of the $\mathrm{HO}$ energy $\hbar \omega$. Circles and triangles are given with the Hamiltonian in Figs. 5(b) and 5(a), respectively. Squares are obtained after the diagonalization of the Hamiltonian in Fig. 5(a) in the $0 \mathrm{p} 0 \mathrm{~h}$ and $1 \mathrm{p} 1 \mathrm{~h}$ space. The dashed curves are obtained with the Op0h HO reference state.

After the diagonalization in the $0 \mathrm{p} 0 \mathrm{~h}$ and $1 \mathrm{p} 1 \mathrm{~h}$ space (green squares) as employed in the earlier framework [21,22,28,29], the $\hbar \omega$ dependence on the results is slightly reduced compared to the UMOA without $S^{(1)}$ (blue triangles). In contrast, the situation drastically changes if the transformation operator $U$ is constructed by the one- and two-body correlation operators, as in the current formalism. The results from the UMOA with $S^{(1)}$ (red circles) are practically $\hbar \omega$ independent both for the energy and radius. This is the main result in this paper: showing that the one-body correlation operator functions well in the UMOA to reduce the $\hbar \omega$ dependence, which is needed to obtain reliable converged results. In the present framework, we do not have to care about the choice of $\hbar \omega$, if the calculations are done in sufficiently large model spaces.

There are additional remarks on the optimum $\hbar \omega$ value in the UMOA. In the CCM [30], the choice of the $\hbar \omega$ values is discussed by comparing the results with and without the one-body correlation operator. In Ref. [30], the authors concluded that the results (calculated without the one-body correlation operator) at $\hbar \omega$ minimizing the ground-state energy agree with the $\hbar \omega$-independent results (calculated with the one-body correlation operator). This statement is confirmed by the present UMOA calculations. Our results for energies with (circles) and without (triangles) the $S^{(1)}$ operator are very close to each other around the optimum $\hbar \omega$ value of $\hbar \omega=28 \mathrm{MeV}$ in Fig 7. There is, however, a word of caution about the choice of the $\hbar \omega$ value for the results after the diagonalization in the earlier UMOA. In our previous investigations [28,29], the ground-state energies and charge radii of doubly magic nuclei have been calculated in the UMOA without $S^{(1)}$. Then, the results converge with respect to the model-space size, while the $\hbar \omega$ dependence of the results, especially for charge radii, is not negligible. To determine accurately the radii, we have taken the results after the diagonalization at $\hbar \omega$ minimizing the ground-state energy, corresponding to the square symbol at $\hbar \omega=20 \mathrm{MeV}$ in Fig. 7. Since we do not confirm the agreement of the results with the diagonalization (squares) and with $S^{(1)}$ (circles) at $\hbar \omega=20 \mathrm{MeV}$ in Fig. 7, such an estimation would not be valid after the diagonalization. In the present framework, however, we do not have to care about the choice of $\hbar \omega$, because of the weak $\hbar \omega$ dependence of the results.

After glancing over the effect of the one-body correlation operator, we next discuss how the $\hbar \omega$ dependence of the results is reduced by introducing the $S^{(1)}$ operator. To look closer at the role of the one-body correlation operator, it is convenient to decompose the expectation value into the contributions from each cluster term. In the case of the ground-state energy, it can be decomposed into energies from the one-body kinetic term $\widetilde{t}_{1}$, two-body cluster term $\widetilde{v}_{12}$, and three-body cluster term $\widetilde{v}_{123}$. Figure 8 describes the energies from each decomposed cluster term. The total, one-body, two-body, and three-body energies of the ${ }^{4} \mathrm{He}$ ground state are shown as functions of $\hbar \omega$ from the top to the bottom in the figure. As a reference, the $\mathrm{HO}$ (dashed line) and HF (dotted) reference states are also drawn. From the figure, one can find that the contribution from the three-body cluster term is smaller by two orders of magnitude than the one- and two-body cluster terms. The cluster expansion in the UMOA converges in the case of the ${ }^{4} \mathrm{He}$ ground-state energy. Also, the $\hbar \omega$ dependence of the energy expectation values is reduced at each order of the cluster expansion.

Now, let us focus on the one-body kinetic energy part (the second panel from the top in Fig. 8). When we ignore $S^{(1)}$ from the beginning $\left(S^{(1)}=0\right),\left\langle t_{1}\right\rangle$ is nothing but the sum of the diagonal component of the matrix $\left\langle a\left|t_{1}\right| a\right\rangle$. Here, $\langle X\rangle$ means the expectation value of an operator $X$ with respect to the $\mathrm{HO}$ $0 \mathrm{p} 0 \mathrm{~h}$ reference state $|\mathrm{HO}\rangle$. In the case of the ${ }^{4} \mathrm{He}$ ground state, one can easily find that $\left\langle t_{1}\right\rangle$ (denoted by the blue triangles in the second panel from the top in Fig. 8) is proportional to $\hbar \omega$ as

$$
\begin{aligned}
\left\langle t_{1}\right\rangle & =\left(1-\frac{1}{A}\right) \sum_{a \leqslant \rho_{F}}\left(2 n_{a}+l_{a}+\frac{3}{2}\right) \frac{\hbar \omega}{2} \\
& =\frac{9}{4} \hbar \omega,
\end{aligned}
$$



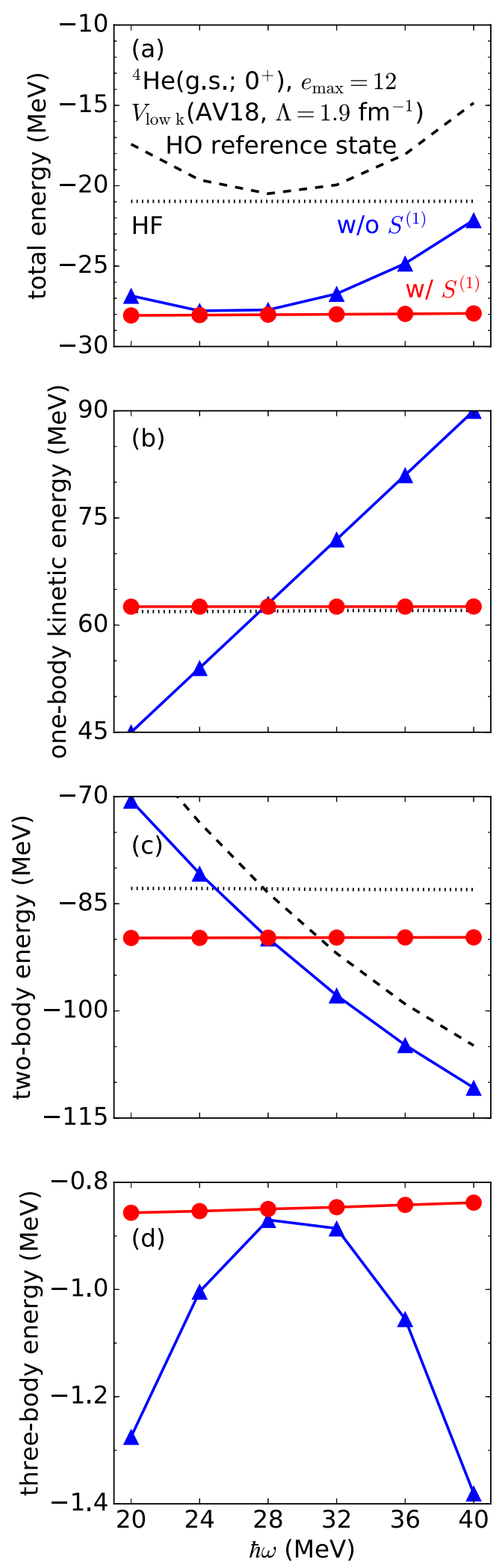

FIG. 8. The total (a), one-body (b), two-body (c), and three-body energies (d) for ${ }^{4} \mathrm{He}$ as functions of $\hbar \omega$ from the top to the bottom. The $N N$ interaction is the $V_{\text {low k }}$ interaction derived from AV18 interaction [38] at $\Lambda=1.9 \mathrm{fm}^{-1}$. Circles (triangles) correspond to the results with (without) $S^{(1)}$. The dashed and dotted lines are for the HO reference and $\mathrm{HF}$ states, respectively. with respect to the HO reference state $(0 s 1 / 2)^{4}$. On the other hand, when $S^{(1)}$ is introduced, the off-diagonal component of the original matrix $\left\langle a\left|t_{1}\right| b\right\rangle(a \neq b)$ contributes to $\left\langle\widetilde{t}_{1}\right\rangle=\left\langle e^{-s_{1}} t_{1} e^{s_{1}}\right\rangle$. As a result, $\left\langle\widetilde{t}_{1}\right\rangle$ becomes practically $\hbar \omega$ independent (as denoted by the red circles in the figure). Also, these are quite close to the HF kinetic energy $\left\langle\mathrm{HF}\left|t_{1}\right| \mathrm{HF}\right\rangle$ (black dotted curve in the figure). Thus, the $S^{(1)}$ operator acts as the generator of the transformation from $|\mathrm{HO}\rangle$ to $|\mathrm{HF}\rangle$ in the UMOA.

Almost the same discussion can be done for the expectation value of the two-body cluster term. The dashed line in the third panel from the top in Fig. 8 corresponds to

$$
\left\langle v_{12}\right\rangle=\frac{1}{2} \sum_{a, b \leqslant \rho_{F}}\left\langle a b\left|v_{12}\right| a b\right\rangle,
$$

which is the sum of the diagonal components of the original two-body matrix elements. As seen in the figure, it shows the monotonic dependence on $\hbar \omega$. This tendency can be understood by assuming a simple $S$-wave two-nucleon potential model such as the contact interaction regularized by the Gaussian with a cutoff momentum $\Lambda_{\delta}$ :

$$
V_{\delta}= \begin{cases}C_{{ }^{1} S_{0}} \exp \left(-\frac{q^{2}+q^{2}}{\Lambda_{\delta}^{2}}\right) & \text { for }{ }^{1} S_{0} \text { channel, } \\ C^{{ }^{3} S_{1}} \exp \left(-\frac{q^{2}+q^{12}}{\Lambda_{\delta}^{2}}\right) & \text { for }{ }^{3} S_{1} \text { channel. }\end{cases}
$$

Here, $q$ and $q^{\prime}$ are the magnitudes of relative momenta for the initial and final states, respectively. The $C_{1} S_{0}$ and $C_{{ }^{3}} S_{1}$ are the low-energy constants for ${ }^{1} S_{0}$ and ${ }^{3} S_{1}$ channels, respectively. Note that only the $S$-wave potentials are enough for the discussion about the $(0 s 1 / 2)^{4}$ single-particle configuration. As discussed in Appendix C, one can obtain

$$
\left\langle V_{\delta}\right\rangle=3 \sqrt{\frac{\pi}{2}}\left(C_{{ } S_{0}}+C_{{ }^{3} S_{1}}\right)\left(\frac{\sqrt{\hbar m \omega} \Lambda_{\delta}^{2}}{m \omega+\hbar \Lambda_{\delta}^{2}}\right)^{3} .
$$

The $\hbar \omega$ dependence is given by the derivative of $\left\langle V_{\delta}\right\rangle$ with respect to $\omega$. As shown in Eq. (C11), the derivative is always negative in our $\hbar \omega$ range from 20 to $40 \mathrm{MeV}$, while the $\mathrm{HF}$ result (dotted curve) shows rather weaker $\hbar \omega$ dependence. Similar behavior can be found in the UMOA with and without $S^{(1)}$ (circles and triangles, respectively). Accordingly, the $\hbar \omega$ dependence is reduced by the effect of $S^{(1)}$.

Like the one- and two-body cluster terms, the $\hbar \omega$ dependence on the three-body cluster term is also reduced, as shown in the bottom panel of Fig. 8. From these considerations, the inclusion of the $S^{(1)}$ operator mitigates considerably the $\hbar \omega$ dependence of the energy expectation value at each order of the cluster expansion. Moreover, the relations between the UMOA with and without $S^{(1)}$ resemble those between the results with the $\mathrm{HF}$ and $\mathrm{HO} 0 \mathrm{p} 0 \mathrm{~h}$ states. Note that, if we ignore $S^{(2)}$ from the beginning, calculated ground-state energies numerically coincide with the HF ground-state energies within a few-keV level. Thus, a constant shift in the two-body energy comes from the $S^{(2)}$ contribution.

To examine more directly the role of $S^{(1)}$, the overlap of wave functions is also investigated. In Fig. 9, we show three squared overlaps obtained with the HF and HO reference states (black dashed line), the UMOA with and without $S^{(1)}$ 

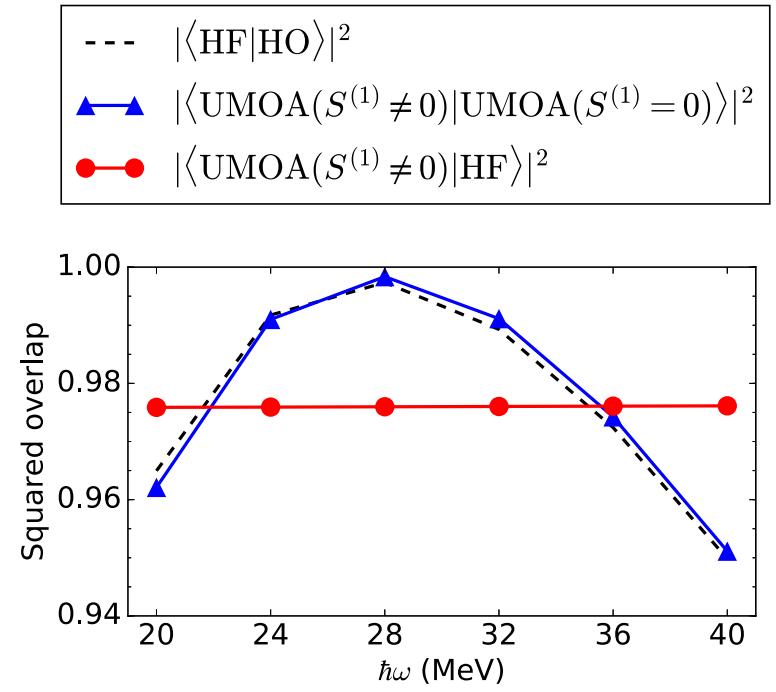

FIG. 9. The squared overlaps between the ground and reference states as functions of $\hbar \omega$. The employed $N N$ interaction is the $V_{\text {low k }}$ interaction derived from AV18 interaction at $\Lambda=1.9 \mathrm{fm}^{-1}$. Circles (triangles) are obtained with the UMOA with $S^{(1)}$ and HF (UMOA without $S^{(1)}$ ) states. The dashed line is given with the HF and HO reference states.

(blue triangles), and the UMOA with $S^{(1)}$ and HF states (red circles). Note that the UMOA results are obtained at $e_{\max }=12$. The squared overlap $|\langle\mathrm{HO} \mid \mathrm{HF}\rangle|^{2}$ (dashed line) indicates the effect of the optimization of single-particle basis states. In Fig. 9, the squared overlaps $|\langle\mathrm{HO} \mid \mathrm{HF}\rangle|^{2}$ (black dashed line) and $\left|\left\langle\operatorname{UMOA}\left(S^{(1)} \neq 0\right) \mid \operatorname{UMOA}\left(S^{(1)}=0\right)\right\rangle\right|^{2}$ (blue triangles) behave in a similar way. Therefore, the role of $S^{(1)}$ is to optimize the single-particle basis states, as expected from the above discussion of the ground-state energy. As a check, we confirm that the overlap $\left|\left\langle\operatorname{UMOA}\left(S^{(1)} \neq 0\right) \mid \mathrm{HF}\right\rangle\right|^{2}$ (red circles) does not depend on the $\hbar \omega$ values.

Further insight can be acquired by looking into the onebody density matrix $\gamma$. The derivation of the one-body density matrix in the UMOA is shown in Appendix B. Using Eq. (B8), we have $\left\langle\widetilde{t}_{1}\right\rangle_{\text {L.O. }}=\operatorname{Tr}\left(\gamma t_{1}\right)$ at the leading order of cluster expansion. Figure 10 shows the density matrices for the $s 1 / 2$ orbital with the $\hbar \omega$ values ranging from 20 to $36 \mathrm{MeV}$ at $e_{\max }=12$. For visibility of the figure, we only show the density matrices up to the components of $\max \left(n_{a}, n_{b}\right)=3$. When the one-body correlation operator is switched off $\left(S^{(1)}=0\right)$, only the component of the density matrix $\gamma_{0 s 1 / 2,0 s 1 / 2}$ is dominant. Then, as shown in Eq. (46), $\left\langle t_{1}\right\rangle$ linearly increases as a function of $\hbar \omega$. In contrast, the off-diagonal elements, especially $\gamma_{0 s 1 / 2,1 s 1 / 2}$, can have large values, when the one-body correlation operator is turned on $\left(S^{(1)} \neq 0\right)$. At $\hbar \omega=20 \mathrm{MeV}, \gamma_{0 s 1 / 2,1 s 1 / 2}$ is positive and increases $\left\langle\widetilde{t}_{1}\right\rangle$. Note that the off-diagonal component of $\left\langle a\left|t_{1}\right| b\right\rangle$ is always positive. On the other hand, $\gamma_{0 s 1 / 2,1 s 1 / 2}$ is negative and decreases $\left\langle\widetilde{t}_{1}\right\rangle$ at $\hbar \omega=36 \mathrm{MeV}$. The contribution from the off-diagonal components to $\left\langle\widetilde{t}_{1}\right\rangle$ balances around $\hbar \omega=28$ $\mathrm{MeV}$, because $\gamma_{0 s 1 / 2,1 s 1 / 2}$ is almost zero there. This finding is consistent with the behavior of one-body energies shown in Fig. 8.
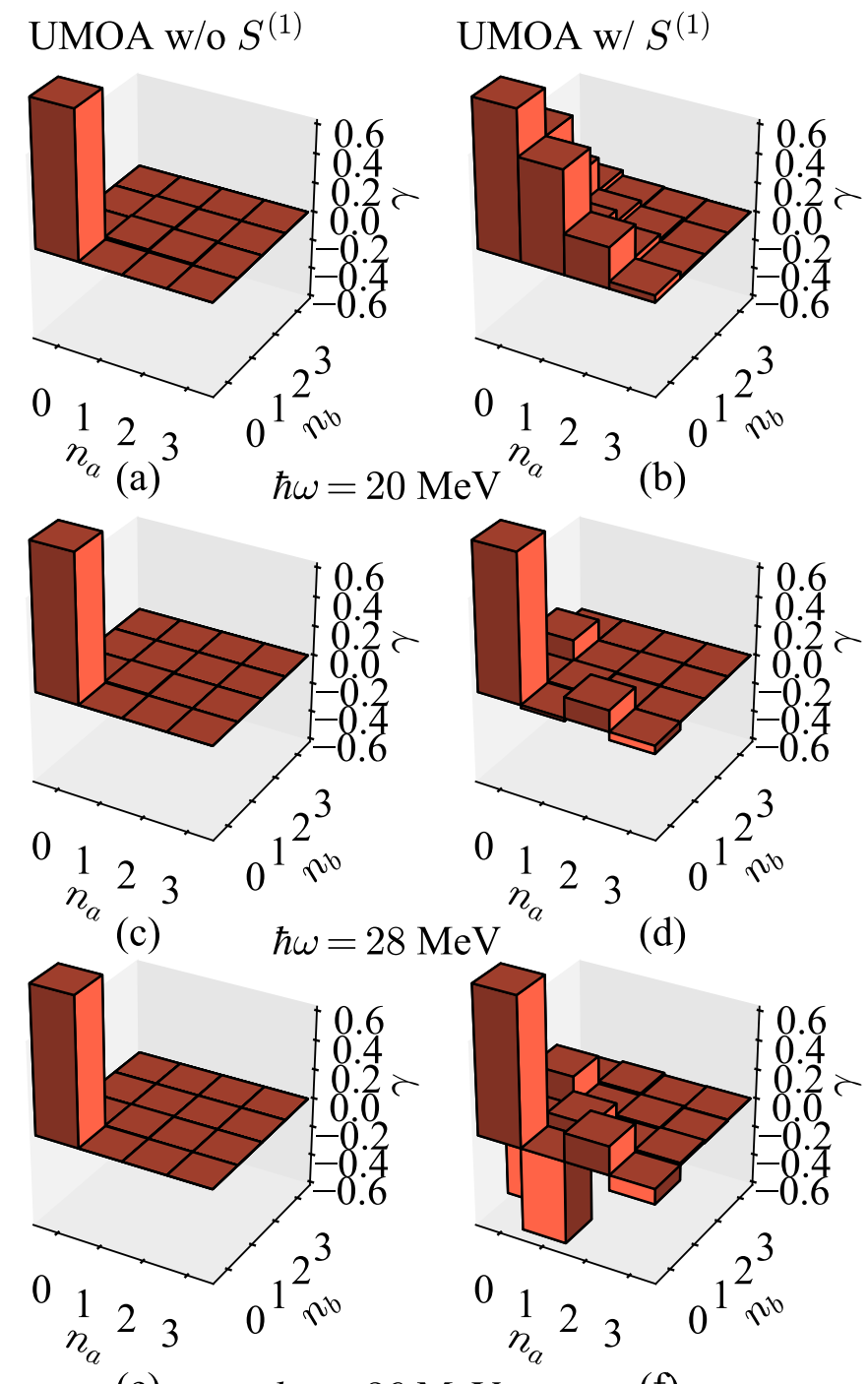

(e)

$$
\hbar \omega=36 \mathrm{MeV}
$$

(d)

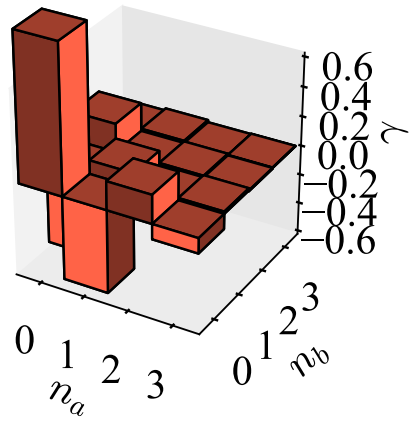

(f)

FIG. 10. One-body density matrix $\gamma$ of $s 1 / 2$ orbitals for ${ }^{4} \mathrm{He}$ calculated without [left; panels (a), (c), and (e)] and with [right; panels (b), (d), and (f)] the one-body correlation operator. The top, middle, and bottom panels are calculated at $e_{\max }=12$ and $\hbar \omega=20$, 28 , and $36 \mathrm{MeV}$, respectively.

Next, we discuss the effects of the one-body correlation operator on the point-nucleon radius. Since the radius operator is dominated by the one-body term, the one-body density matrix concerns more directly the reduction of the $\hbar \omega$ dependence rather than the ground-state energy where the two-body correlations are dominant. As shown in the lower panel in Fig. 7, the point-nucleon radius of the ${ }^{4} \mathrm{He}$ nucleus with respect to the $\mathrm{HO}$ reference state $(0 s 1 / 2)^{4}$ can read

$$
\begin{aligned}
\sqrt{\left\langle r_{1}^{2}\right\rangle} & =\sqrt{\sum_{a} \frac{1}{A}\left(1-\frac{1}{A}\right) \frac{(\hbar c)^{2}}{m c^{2} \hbar \omega}\left(2 n_{a}+l_{a}+\frac{3}{2}\right)} \\
& \simeq 6.8(\hbar \omega)^{-1 / 2}(\mathrm{MeV})^{1 / 2} \mathrm{fm} .
\end{aligned}
$$

From the observation that the off-diagonal components of the kinetic and radius one-body operators contribute oppositely, 
TABLE I. Calculated ground-state energy of ${ }^{4} \mathrm{He}$ with the decomposition of each cluster term. The results in the section named " $V_{\text {low k }}$ " are calculated with the $V_{\text {low k }}$ interaction derived from AV18 interaction with the sharp cutoff $\Lambda=1.9 \mathrm{fm}^{-1}$. Also, the results in section " $V_{\mathrm{SRG}}$ " are obtained with the SRG transformed chiral $\mathrm{N}^{3} \mathrm{LO}$ $N N$ interaction at $\lambda_{\mathrm{SRG}}=2.0 \mathrm{fm}^{-1}$. All the energies are in MeV. See text for details.

\begin{tabular}{llllll}
\hline \hline & Method & $E^{1 \mathrm{BC}}$ & $E^{2 \mathrm{BC}}$ & $E^{3 \mathrm{BC}}$ & \multicolumn{1}{c}{$E_{\text {g.s. }}$} \\
\hline$V_{\text {low k }}$ & UMOA & 62.60 & -89.84 & -0.86 & -28.10 \\
& CCSD [2] & & & & -28.9 \\
& FY [2,41] & & & & $-29.19(5)$ \\
$V_{\text {SRG }}$ & UMOA & 53.50 & -80.47 & -0.76 & -27.73 \\
& IT-NCSM [42] & & & & $-28.25(1)$ \\
Exp. [43] & & & & & -28.30 \\
\hline \hline
\end{tabular}

we can expect that the radius decreases (increases) in smaller (larger) $\hbar \omega$ regions across $\hbar \omega \simeq 28 \mathrm{MeV}$ compared to the $\mathrm{HO}$ reference state, Eq. (50). This is consistent with the radii shown in the lower panel of Fig. 7.

\section{B. Comparison with the other $a b$ initio results}

After discussing how the introduction of the one-body correlation operator in the UMOA works well, in this section, we compare the present results with those from the other $a b$ inito calculations. For this purpose, we employ the $V_{\text {low } \mathrm{k}}$ potential derived from AV18 two-nucleon interaction at $\Lambda=$ $1.9 \mathrm{fm}^{-1}$ throughout the comparison. In addition, we also employ the SRG-transformed chiral $\mathrm{N}^{3} \mathrm{LO} N N$ interaction [40] with the momentum cutoff $\lambda_{\mathrm{SRG}}=2.0 \mathrm{fm}^{-1}$, which is widely used in recent $a b$ initio calculations.

The results of the ${ }^{4} \mathrm{He}$ ground-state energy are summarized in Table I with comparison to the other $a b$ inito results. In Table I, the calculated energies are shown with the decomposition of the one-body cluster (kinetic) term, $E^{1 \mathrm{BC}}$, two-body cluster (interaction) term, $E^{2 \mathrm{BC}}$, the three-body cluster term, $E^{3 \mathrm{BC}}$, and the total energy, $E_{\text {g.s. }}$ for ${ }^{4} \mathrm{He}$, respectively [see Eq. (33)]. All the results are calculated at $e_{\max }=14$ and $\hbar \omega=20 \mathrm{MeV}$. For comparison, the results of the CCSD, FY, importance-truncated no-core shell model (IT-NCSM), and experiment are taken from Refs. [2,41-43]. We notice that the convergence with respect to the model-space size is confirmed. The difference between $e_{\max }=12$ and 14 results is less than the order of $10 \mathrm{keV}$ both for the $V_{\text {low } \mathrm{k}}$ and SRG transformed interactions. As shown in Table I, the contribution of the three-body cluster term, $E^{3 \mathrm{BC}}$, is much smaller than those of one- and two-body cluster terms, $E^{1 \mathrm{BC}}$ and $E^{2 \mathrm{BC}}$, respectively. Therefore, our result for the energy practically converges with respect to the cluster expansion. Moreover, the present result for the total energy, $E_{\text {g.s. }}$, is reasonably close to the other $a b$ initio calculation energies. The difference between them is comparable to the size of $E^{3 \mathrm{BC}}$. In other words, the contributions of the truncated cluster terms can be approximated by the size of $E^{3 \mathrm{BC}}$ and the uncertainty of our energies can be roughly estimated from $E^{3 \mathrm{BC}}$. In Fig. 11, we also summarize the comparison of various calculated energies.

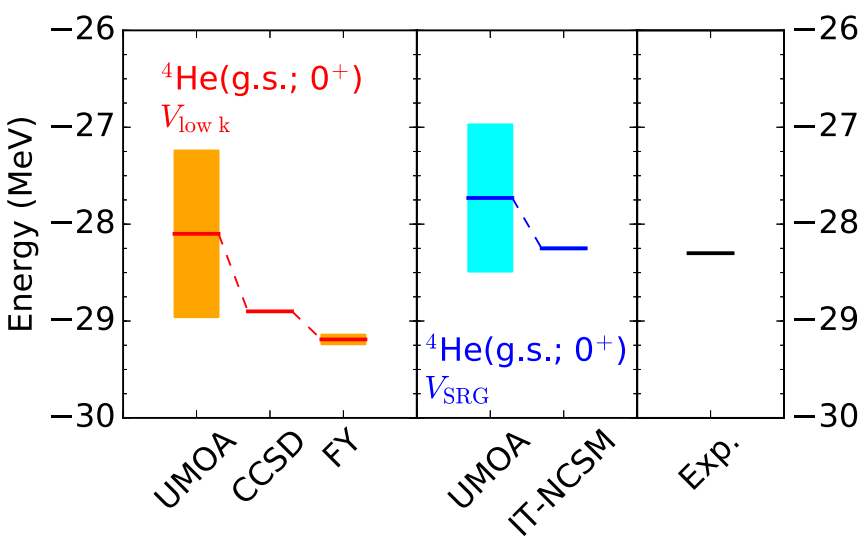

FIG. 11. Comparisons for the ground-state energies of ${ }^{4} \mathrm{He}$ in the UMOA and in the other $a b$ initio calculations. The displayed UMOA results are obtained at $e_{\max }=14$ and $\hbar \omega=20 \mathrm{MeV}$. The error bands of the UMOA energies are estimated from the size of the three-body cluster term corrections. The CCSD, FY, importance-truncated nocore shell model (IT-NCSM), and experimental results are taken from Refs. [2,41-43].

The error bands of the UMOA energies are estimated from the size of the three-body cluster term corrections.

Our results for point-nucleon radius of ${ }^{4} \mathrm{He}$ are listed in Table II. It summarizes the calculated point-nucleon radii with the "one-body cluster term" $\sqrt{\widetilde{r}^{2(1 \mathrm{BC})}}$, "two-body cluster term" $\sqrt{\widetilde{r}^{2(2 \mathrm{BC})}}$, and the "one- and two-body cluster term" $\sqrt{\widetilde{r}^{2}}$, respectively [see Eq. (45)]. Note that $\sqrt{\widetilde{r}^{2}} \neq \sqrt{\widetilde{r}^{2(1 \mathrm{BC})}}+$ $\sqrt{\widetilde{r}^{2(2 \mathrm{BC})}}$, and $\widetilde{r}^{2}=\widetilde{r}^{2(1 \mathrm{BC})}+\widetilde{r}^{2(2 \mathrm{BC})}$. The calculation setup is same as in Table I. The experimental value is take from Ref. [44]. According to Table II, the contribution from the one-body cluster term, $\sqrt{\widetilde{r}^{2(1 \mathrm{BC})}}$, is dominant. Since the effects of the higher-body cluster terms can be expected to be smaller than those of the one-body cluster term, the cluster expansion works well for the radius operator, the same as in the case of the ground-state energy. Moreover, the present result for point-proton radius, $1.41 \mathrm{fm}$, with the SRG softened N3LO chiral EFT $N N$ interaction [40] with $\lambda_{\mathrm{SRG}}=2.0 \mathrm{fm}^{-1}$ is consistent with the in-medium SRG results with the same interaction [45]. Note that the calculated radius is much smaller than the experimental radius, $1.49 \mathrm{fm}$. This observation is also true for the other $a b$ initio calculations with $N N$ interactions only and is consistent with the obtained larger binding energy compared to the experimental binding energy. From these demonstrations, we can conclude that the UMOA with the aid of the one-body correlation operator gives fully microscopic results as well as the other ab initio approaches, at least in the ground-state properties of the ${ }^{4} \mathrm{He}$ nucleus.

TABLE II. Calculated point-nucleon radius of ${ }^{4} \mathrm{He}$ with the decomposition of each cluster term. See text for details.

\begin{tabular}{llcl}
\hline \hline & $\sqrt{\widetilde{r}^{2(1 \mathrm{BC})}}$ & $\sqrt{\widetilde{r}^{2(2 \mathrm{BC})}}$ & \multicolumn{1}{c}{$\sqrt{\widetilde{r}^{2}}$} \\
\hline$V_{\text {low k }}$ & 1.30 & 0.20 & 1.32 \\
$V_{\text {SRG }}$ & 1.40 & 0.13 & 1.41 \\
Exp. [44] & & & $1.49(3)$ \\
\hline \hline
\end{tabular}




\section{SUMMARY}

We extend the former formalism of the UMOA accommodating only two-body correlations by including additionally the one-body correlation operator. As a demonstration, we have carried out numerical calculations of the ground-state energy and point-nucleon radius for the ${ }^{4} \mathrm{He}$ nucleus. We can successfully reduce the $\hbar \omega$ dependence on calculated observables which existed in the earlier UMOA. The present results for converged ground-state energy and radius are reasonably close to the results from the other $a b$ initio calculations with the same $N N$ interactions. We find that the hierarchy of the cluster expansion is preserved and the $\hbar \omega$ dependence can be removed, order by order, from the cluster expansion. The decoupling of the $1 \mathrm{p} 1 \mathrm{~h}$ excitations from the $0 \mathrm{p} 0 \mathrm{~h}$ reference state is related to the HF mean field to absorb the $\hbar \omega$ dependence. The origin of the $\hbar \omega$ dependence in the former UMOA has also been discussed qualitatively. Concerning the behavior of the total energy as a function of $\hbar \omega$, the one-body kinetic term totally entails the positive slope, while the two-body potential term causes the negative slope, which results in the parabolic shape of the total energy. The above discussion about the one-body kinetic energy can also explain the negative slope of the radius as a function of $\hbar \omega$.

In this study, we only employ two-nucleon interactions without genuine and/or induced three-nucleon interactions. For quantitative comparison with experimental data, the introduction of three-body forces is requisite. For this purpose, the systematic improvement of the UMOA is necessary. The next step to be done for this direction is to explicitly deal with the three-body cluster term and to introduce the three-body correlation operator. These implementations in the UMOA described above will open the way toward a fully microscopic description of nuclear structure consistent with the other $a b$ initio methods. As for the physical application, in this paper, we only focus on the ground-state property of the lightest doubly closed nucleus, ${ }^{4} \mathrm{He}$, as a test case of the implementation of the one-body correlation operator in the UMOA. Application to heavier nuclei such as ${ }^{16} \mathrm{O}$ and ${ }^{40} \mathrm{Ca}$ is also interesting. The extension to the excited states can also be done, for example, with the method introduced in Ref. [21]. Furthermore, the UMOA can be applied to the nuclear structure of open-shell nuclei and gives information about the one-nucleon separation energy $[21,22]$. These studies are now ongoing, and the results will be reported elsewhere in future publications.

\section{ACKNOWLEDGMENTS}

The authors thank M. Kohno, K. Suzuki, H. Kumagai, S. Fujii, N. Shimizu, B. R. Barrett, P. Navrátil, and S. R. Stroberg for many useful discussions. This work was supported in part by JSPS KAKENHI Grant No. JP16J05707 and by the Program for Leading Graduate Schools, MEXT, Japan. This work was also supported in part by MEXT SPIRE and JICFuS (Projects No. hp160211 and No. hp170230) and the CNS-RIKEN joint project for large-scale nuclear structure calculations.

\section{APPENDIX A: DECOUPLING EQUATIONS}

In this appendix, we briefly explain how to determine the correlation operators used in the UMOA [34]. Owing to the definition of the unitary operator $U$ as shown in Eq. (8), $\exp S^{(n)}$ appears only in the $n$-body and higher body cluster terms and does not affect cluster terms lower than $n$-body clusters [for instance, two- and higher many-body correlation operators do not show up in the one-body cluster terms of Eqs. (14) and (17)]. Therefore, we can sequentially solve correlation operators in the order from the one- to $A$-body cluster terms. In the following, we focus on the determination of the $n$ body correlation operator $S^{(n)}$, provided that the correlation operators with the rank lower than $n, S^{(1)}, S^{(2)}, \ldots, S^{(n-1)}$, are already obtained. The correlation operator $S^{(n)}$ is determined so that $\widetilde{H}^{(n)}$ has no matrix elements between the $0 \mathrm{p} 0 \mathrm{~h}$ and $n$ p $n$ h states. For this purpose, we define the operators $P^{(n)}$ and $Q^{(n)}$ projecting onto the space of $n$ particles occupying the orbits below and above the Fermi level, respectively. Then, the decoupling condition between the target space $P^{(n)}$ and its complement $Q^{(n)}$ can be described as

$$
Q^{(n)} \widetilde{H}^{(n)} P^{(n)}=P^{(n)} \widetilde{H}^{(n)} Q^{(n)}=0 .
$$

Equation (A1) can be rewritten as

$$
Q^{(n)} e^{-S^{(n)}} \widetilde{H}^{\prime(n)} e^{S^{(n)}} P^{(n)}=P^{(n)} e^{-S^{(n)}} \widetilde{H}^{\prime(n)} e^{S^{(n)}} Q^{(n)}=0
$$

with

$$
\begin{aligned}
\widetilde{H}^{\prime(n)}= & e^{-\left(\sum_{i_{1}<\cdots<i_{n-1}}^{n} s_{i_{1} \cdots i_{n-1}}\right)} \ldots e^{-\left(\sum_{i}^{n} s_{i}\right)} \\
& \times\left(\sum_{i}^{n} h_{i}+\sum_{k=2}^{n} \sum_{i_{1}<\cdots<i_{k}}^{n} v_{i_{1} \ldots i_{k}}+\sum_{k=2}^{n} \sum_{i_{1}<\cdots<i_{k}}^{n} w_{i_{1} \ldots i_{k}}\right) \\
& \times e^{\sum_{i}^{n} s_{i}} \ldots e^{\sum_{i_{1}<\cdots<i_{n-1}}^{n} s_{i_{1} \ldots i_{n-1}}} .
\end{aligned}
$$

For deriving Eq. (A2) from Eq. (A1), one can use the fact that

$$
\begin{array}{r}
Q^{(n)}\left(\sum_{i_{n}<\cdots<i_{n-1}}^{A} \widetilde{w}_{i_{1} \ldots i_{n-1}}\right) P^{(n)}=0, \\
Q^{(n)}\left(\sum_{i}^{n} \widetilde{h}_{i}+\sum_{k=2}^{n-1} \sum_{i_{1}<\cdots<i_{k}}^{n} \widetilde{v}_{i_{1} \ldots i_{k}}\right) P^{(n)}=0,
\end{array}
$$

in Eqs. (16) and (19), since $P^{(n)}$ and $Q^{(n)}$ have no common states. Thus, $\widetilde{H}^{(n)}$ in Eq. (A1) can be factorized into $\widetilde{H}^{\prime(n)}$ and $e^{S^{(n)}} . \widetilde{H}^{\prime(n)}$ can be calculated in a straightforward way, and the problem can be cast into the determination of $S^{(n)}$. According to Refs. [32-34], it is known that one of the solutions can be expressed as

$$
S^{(n)}=\operatorname{arctanh}\left(\omega^{(n)}-\omega^{(n) \dagger}\right)
$$

with the wave operator for $n$-particle states,

$$
\omega^{(n)}=\sum_{k=1}^{d} Q^{(n)}\left|\psi_{k}^{(n)}\right\rangle\left\langle\widetilde{\phi}_{k}^{(n)}\right| P^{(n)} .
$$

Here, $d$ is the dimension of the $P^{(n)}$ space and the biorthogonal state $\left\langle\widetilde{\phi}_{k}^{(n)}\right|$ of $\left|\phi_{k}^{(n)}\right\rangle=P^{(n)}\left|\psi_{k}^{(n)}\right\rangle$ is defined to satisfy the following biorthonormal condition

$$
\left\langle\widetilde{\phi}_{k}^{(n)} \mid \phi_{l}^{(n)}\right\rangle=\delta_{k l}
$$


In Eq. (A7), $\left|\psi_{k}^{(n)}\right\rangle$ is an eigenvector of the $n$-body Schrödinger equation in the $P^{(n)}+Q^{(n)}$ space,

$$
\left(P^{(n)}+Q^{(n)}\right) \tilde{H}^{(n)}\left(P^{(n)}+Q^{(n)}\right)\left|\psi_{k}^{(n)}\right\rangle=E_{k}\left|\psi_{k}^{(n)}\right\rangle,
$$

where $E_{k}$ is the $k$ th eigenvalue. Moreover, $\exp S^{(n)}$ can also be expressed in terms of $\omega^{(n)}$ [20],

$$
e^{S^{(n)}}=\left(1+\omega^{(n)}-\omega^{(n) \dagger}\right)\left(1+\omega^{(n) \dagger} \omega^{(n)}+\omega^{(n)} \omega^{(n) \dagger}\right)^{-1 / 2} .
$$

Note that the solution of $\omega^{(n)}$ depends on the choice of a set of $d$ eigenstates. In the present work, we choose $d$ eigenstates having the largest overlap with the reference state. This choice is reasonable as long as we consider only the ground state.

\section{APPENDIX B: ONE-BODY DENSITY MATRIX}

Here, we summarize how to compute the one-body density matrix in the UMOA. As found in the usual textbooks (see, for example, Ref. [46]), the one-body density-matrix element $\gamma_{b a}$ is defined as

$$
\gamma_{b a} \equiv\left\langle\Psi\left|n^{a b}\right| \Psi\right\rangle=\left\langle\Psi\left|c_{a}^{\dagger} c_{b}\right| \Psi\right\rangle
$$

with the one-body operator $n^{a b}$ concerning the single-particle states labeled by $a$ and $b$. The one-body operator $n^{a b}$ can be transformed as

$$
\begin{aligned}
\gamma_{b a} & =\left\langle\Phi\left|U^{\dagger} n^{a b} U\right| \Phi\right\rangle \\
& =\left\langle\Phi\left|\widetilde{n}^{a b}\right| \Phi\right\rangle
\end{aligned}
$$

with the unitary-transformed one-body operator $\widetilde{n}^{a b}=$ $U^{\dagger} n^{a b} U$ and the reference state $|\Phi\rangle$. Like the Hamiltonian and radius operator discussed in the text, we apply the cluster expansion to this transformed one-body operator,

$$
\widetilde{n}^{a b}=\widetilde{n}^{a b(1)}+\widetilde{n}^{a b(2)}+\cdots .
$$

Here, one- and two-body cluster terms are defined as

$$
\tilde{n}^{a b(1)}=\sum_{i} \widetilde{n}_{i}^{a b}, \quad \widetilde{n}^{a b(2)}=\sum_{i<j} \widetilde{n}_{i j}^{a b}
$$

with

$$
\begin{aligned}
& \tilde{n}_{1}^{a b}=e^{-s_{1}} n_{1}^{a b} e^{s_{1}}, \\
& \widetilde{n}_{12}^{a b}=e^{-s_{12}}\left(\widetilde{n}_{1}^{a b}+\widetilde{n}_{2}^{a b}\right) e^{s_{12}}-\left(\widetilde{n}_{1}^{a b}+\widetilde{n}_{2}^{a b}\right) .
\end{aligned}
$$

Then, the matrix element $\gamma_{b a}$ can also be expanded as

$$
\gamma_{b a}=\sum_{\lambda \leqslant \rho_{F}}\left\langle\lambda\left|\widetilde{n}_{1}^{a b}\right| \lambda\right\rangle+\frac{1}{2} \sum_{\lambda \mu \leqslant \rho_{F}}\left\langle\lambda \mu\left|\widetilde{n}_{12}^{a b}\right| \lambda \mu\right\rangle+\cdots .
$$

In the actual UMOA calculations, we keep the terms up to the two-body clusters. With the aid of the one-body density matrix, the expectation value for one-body operators, $O=$ $\sum_{a b}\langle a|o| b\rangle c_{a}^{\dagger} c_{b}$, can also be obtained by

$$
\begin{aligned}
\langle\Psi|O| \Psi\rangle & =\sum_{a b}\langle a|o| b\rangle\left\langle\Psi\left|c_{a}^{\dagger} c_{b}\right| \Psi\right\rangle=\sum_{a b} o_{a b} \gamma_{b a} \\
& =\operatorname{Tr}(o \gamma)
\end{aligned}
$$

\section{APPENDIX C: EXPECTATION VALUE OF A CONTACT INTERACTION WITH RESPECT TO THE $(0 s 1 / 2)^{4}$ CONFIGURATION}

In this appendix, we derive the expectation value of the contact interaction $V_{\delta}$ introduced in Eq. (48). It is given by the normal ordered zero-body term with respect to the $(0 s 1 / 2)^{4}$ configuration:

$$
\left\langle V_{\delta}\right\rangle=\sum_{J T}(2 J+1)(2 T+1)\left\langle J T\left|V_{\delta}\right| J T\right\rangle
$$

with the $J T$-coupled two-body matrix element $\left\langle J T\left|V_{\delta}\right| J T\right\rangle$. Note that $|J T\rangle$ means the $(0 s 1 / 2)^{2}$ two-nucleon state with the total angular momentum $J$ and total isospin $T$. Also $J+T$ has to be odd integer, because of the antisymmetrization of two-nucleon state. To obtain the expectation value $\left\langle V_{\delta}\right\rangle$, all the terms we need are $\left\langle 10\left|V_{\delta}\right| 10\right\rangle$ and $\left\langle 01\left|V_{\delta}\right| 01\right\rangle$. When we apply the Talmi-Moshinsky transformation formula, they are expressed as

$$
\begin{aligned}
& \left\langle 10\left|V_{\delta}\right| 10\right\rangle=\left\langle\alpha\left|V_{\delta}\right| \alpha\right\rangle, \\
& \left\langle 01\left|V_{\delta}\right| 01\right\rangle=\left\langle\beta\left|V_{\delta}\right| \beta\right\rangle
\end{aligned}
$$

with the two-nucleon states $|\alpha\rangle$ and $|\beta\rangle$ in the relative coordinate. Here, $|\alpha\rangle \quad(|\beta\rangle)$ has the quantum numbers $\quad\left(n, l_{\text {rel }}, S, J_{\text {rel }}, T\right)=(0,0,0,0,1) \quad\left[\left(n, l_{\text {rel }}, S, J_{\text {rel }}, T\right)=\right.$ $(0,0,1,1,0)]$, where $n, l_{\text {rel }}, S, J_{\text {rel }}$, and $T$ are the nodal quantum number, relative orbital angular momentum, total spin, relative total angular momentum, and total isospin for the two-nucleon system, respectively. Since the HO radial wave function with $n=0$ and $l_{\text {rel }}=0$ is just the Gaussian, these expectation values are written as

$$
\begin{aligned}
& \left\langle\alpha\left|V_{\delta}\right| \alpha\right\rangle=C_{{ }^{1} S_{0}} \frac{8 \sqrt{2}}{\sqrt{\pi}}\left(\frac{\hbar}{m \omega}\right)^{3 / 2} \Lambda_{\delta}^{6} I^{2}\left(\omega, \Lambda_{\delta}\right), \\
& \left\langle\beta\left|V_{\delta}\right| \beta\right\rangle=C_{{ }^{3} S_{1}} \frac{8 \sqrt{2}}{\sqrt{\pi}}\left(\frac{\hbar}{m \omega}\right)^{3 / 2} \Lambda_{\delta}^{6} I^{2}\left(\omega, \Lambda_{\delta}\right)
\end{aligned}
$$

with the integration

$$
I\left(\omega, \Lambda_{\delta}\right)=\int_{0}^{\infty} d x x^{2} \exp \left[-\left(\frac{\hbar \Lambda_{\delta}^{2}}{m \omega}+1\right) x^{2}\right] .
$$

This integration can be done analytically and reads

$$
I\left(\omega, \Lambda_{\delta}\right)=\frac{\sqrt{\pi}}{4}\left(\frac{m \omega}{m \omega+\hbar \Lambda_{\delta}^{2}}\right)^{3 / 2} .
$$

Substituting this result into Eqs. (C4) and (C5), one can get

$$
\begin{aligned}
& \left\langle\alpha\left|V_{\delta}\right| \alpha\right\rangle=C^{{ }_{1}} S_{0} \sqrt{\frac{\pi}{2}}\left(\frac{\sqrt{\hbar m \omega} \Lambda_{\delta}^{2}}{m \omega+\hbar \Lambda_{\delta}^{2}}\right)^{3}, \\
& \left\langle\beta\left|V_{\delta}\right| \beta\right\rangle=C^{3} S_{1} \sqrt{\frac{\pi}{2}}\left(\frac{\sqrt{\hbar m \omega} \Lambda_{\delta}^{2}}{m \omega+\hbar \Lambda_{\delta}^{2}}\right)^{3} .
\end{aligned}
$$

Therefore, the expectation value can be obtained as

$$
\left\langle V_{\delta}\right\rangle=3 \sqrt{\frac{\pi}{2}}\left(C_{{ }^{1} S_{0}}+C^{3} S_{1}\right)\left(\frac{\sqrt{\hbar m \omega} \Lambda_{\delta}^{2}}{m \omega+\hbar \Lambda_{\delta}^{2}}\right)^{3} .
$$


Since the $S$-wave scattering phase shift analysis implies that the $N N$ interaction is attractive at low energies, the low-energy constants $C_{1} S_{0}$ and $C_{3} S_{1}$ are usually negative values. Then, the sign of $\left\langle V_{\delta}\right\rangle$ is also negative. To investigate the $\omega$ dependence of $\left\langle V_{\delta}\right\rangle$, let us take the derivative of $\left\langle V_{\delta}\right\rangle$ with respect to $\omega$. After the straightforward calculation, it is

$\frac{d\left\langle V_{\delta}\right\rangle}{d \omega}=\frac{3}{2}\left\langle V_{\delta}\right\rangle \frac{m \Lambda_{\delta}^{2}}{m \omega+\hbar \Lambda_{\delta}^{2}}\left(\sqrt{\frac{\hbar}{m \omega}}+\frac{1}{\Lambda_{\delta}}\right)\left(\sqrt{\frac{\hbar}{m \omega}}-\frac{1}{\Lambda_{\delta}}\right)$.

(C11)
From Eq. (C11), it is found that the sign of the derivative is negative (positive) for $\hbar \omega<\hbar^{2} \Lambda_{\delta}^{2} / m\left(\hbar \omega>\hbar^{2} \Lambda_{\delta}^{2} / m\right)$. Thus, $\left\langle V_{\delta}\right\rangle$ has a minimum at $\hbar \omega=\hbar^{2} \Lambda_{\delta}^{2} / m$. Since $\hbar^{2} \Lambda_{\delta}^{2} / m$ specifies the energy scale of the $N N$ interaction and is roughly the order of, at least, the pion mass, this minimum is far from the $\hbar \omega$ values of $20-40 \mathrm{MeV}$ in the present work. Therefore, the $\hbar \omega$ dependence can be regarded as monotonically decreasing in such a $\hbar \omega$ range.
[1] M. Włoch, D. J. Dean, J. R. Gour, M. Hjorth-Jensen, K. Kowalski, T. Papenbrock, and P. Piecuch, Phys. Rev. Lett. 94, 212501 (2005).

[2] G. Hagen, D. J. Dean, M. Hjorth-Jensen, T. Papenbrock, and A. Schwenk, Phys. Rev. C 76, 044305 (2007).

[3] G. Hagen, T. Papenbrock, D. J. Dean, and M. Hjorth-Jensen, Phys. Rev. Lett. 101, 092502 (2008).

[4] G. Hagen, T. Papenbrock, D. J. Dean, and M. Hjorth-Jensen, Phys. Rev. C 82, 034330 (2010).

[5] G. Hagen, M. Hjorth-Jensen, G. R. Jansen, R. Machleidt, and T. Papenbrock, Phys. Rev. Lett. 108, 242501 (2012).

[6] G. Hagen, M. Hjorth-Jensen, G. R. Jansen, R. Machleidt, and T. Papenbrock, Phys. Rev. Lett. 109, 032502 (2012).

[7] S. Binder, J. Langhammer, A. Calci, P. Navrátil, and R. Roth, Phys. Rev. C 87, 021303(R) (2013).

[8] G. Hagen, T. Papenbrock, M. Hjorth-Jensen, and D. J. Dean, Rept. Prog. Phys. 77, 096302 (2014), and references therein.

[9] S. Binder, J. Langhammer, A. Calci, and R. Roth, Phys. Lett. B 736, 119 (2014).

[10] H. Hergert, S. K. Bogner, S. Binder, A. Calci, J. Langhammer, R. Roth, and A. Schwenk, Phys. Rev. C 87, 034307 (2013).

[11] H. Hergert, S. Binder, A. Calci, and J. Langhammer, and R. Roth, Phys. Rev. Lett. 110, 242501 (2013).

[12] H. Hergert, S. K. Bogner, T. D. Morris, S. Binder, A. Calci, J. Langhammer, and R. Roth, Phys. Rev. C 90, 041302(R) (2014).

[13] C. Barbieri and M. Hjorth-Jensen, Phys. Rev. C 79, 064313 (2009).

[14] A. Cipollone, C. Barbieri, and P. Navrátil, Phys. Rev. Lett. 111, 062501 (2013).

[15] V. Somà, A. Cipollone, C. Barbieri, P. Navrátil, and T. Duguet, Phys. Rev. C 89, 061301(R) (2014).

[16] G. Hagen, A. Ekström, C. Forssén, G. R. Jansen, W. Nazarewicz, T. Papenbrock, K. A. Wendt, S. Bacca, N. Barnea, B. Carlsson et al., Nat. Phys. 12, 186 (2016).

[17] S. R. Stroberg, H. Hergert, J. D. Holt, S. K. Bogner, and A. Schwenk, Phys. Rev. C 93, 051301 (2016).

[18] K. Tsukiyama, S. K. Bogner, and A. Schwenk, Phys. Rev. C 85, 061304 (2012).

[19] S. K. Bogner, H. Hergert, J. D. Holt, A. Schwenk, S. Binder, A. Calci, J. Langhammer, and R. Roth, Phys. Rev. Lett. 113, 142501 (2014).

[20] K. Suzuki and R. Okamoto, Prog. Thoer. Phys. 92, 1045 (1994).

[21] S. Fujii, R. Okamoto, and K. Suzuki, Phys. Rev. C 69, 034328 (2004).
[22] S. Fujii, R. Okamoto, and K. Suzuki, Phys. Rev. Lett. 103, 182501 (2009).

[23] J. DA. Providência and C. M. Shakin, Ann. Phys. 30, 95 (1964).

[24] C. M. Shakin and Y. R. Waghmare, Phys. Rev. 161, 1006 (1967); 161, 1015 (1967).

[25] K. Suzuki and R. Okamoto, Prog. Theor. Phys. 75, 1388 (1986); 76, 127 (1986).

[26] K. Suzuki, R. Okamoto, and H. Kumagai, Phys. Rev. C 36, 804 (1987).

[27] K. Suzuki, Prog. Theor. Phys. 79, 330 (1988).

[28] T. Miyagi, T. Abe, R. Okamoto, and T. Otsuka, JPS Conf. Proc. 6, 030037 (2015).

[29] T. Miyagi, T. Abe, R. Okamoto, and T. Otsuka, Prog. Theor. Exp. Phys. 2015, $041 \mathrm{D} 01$ (2015).

[30] M. Kohno and R. Okamoto, Phys. Rev. C 86, 014317 (2012).

[31] K. Suzuki, Prog. Theor. Phys. 87, 937 (1992).

[32] I. Shavitt and L. T. Redmon, J. Chem. Phys. 73, 5711 (1980).

[33] P. Westhaus, Int. J. Quantum Chem. 20, 1243 (1981).

[34] K. Suzuki, Prog. Theor. Phys. 68, 246 (1982).

[35] E. Schiller, H. Müther, and P. Czerski, Phys. Rev. C 59, 2934 (1999); 60, 059901(E) (1999).

[36] K. Suzuki, R. Okamoto, M. Kohno, and S. Nagata, Nucl. Phys. A 665, 92 (2000).

[37] G. Baardsen, A. Ekström, G. Hagen, and M. Hjorth-Jensen, Phys. Rev. C 88, 054312 (2013).

[38] R. B. Wiringa, V. G. J. Stoks, and R. Schiavilla, Phys. Rev. C 51, 38 (1995).

[39] R. Machleidt, Phys. Rev. C 63, 024001 (2001).

[40] D. R. Entem and R. Machleidt, Phys. Rev. C 68, 041001 (2003).

[41] A. Nogga, S. K. Bogner, and A. Schwenk, Phys. Rev. C 70, 061002 (2004).

[42] R. Roth, J. Langhammer, A. Calci, S. Binder, and P. Navratil, Phys. Rev. Lett. 107, 072501 (2011).

[43] M. Wang, G. Audi, A. H. Wapstra, F. G. Kondev, M. MacCormick, X. Xu, and B. Pfeiffer, Chin. Phys. C 36, 1603 (2012).

[44] G. D. Alkhazov, M. N. Andronenko, A. V. Dobrovolsky, P. Egelhof, G. E. Gavrilov, H. Geissel, H. Irnich, A. V. Khanzadeev, G. A. Korolev, A. A. Lobodenko et al., Phys. Rev. Lett. 78, 2313 (1997).

[45] H. Hergert, S. K. Bogner, T. D. Morris, A. Schwenk, and K. Tsukiyama, Phys. Rep. 621, 165 (2016).

[46] P. Ring and P. Schuck, The Nuclear Many-Body Problem, 3rd ed. (Springer, Berlin, 1980). 